\title{
Four lepton production and the accuracy of QED FSR
}

\author{
Christian Gütschow ${ }^{1, \mathrm{a}}$, Marek Schönherr ${ }^{2, \mathrm{~b}}$ \\ ${ }^{1}$ Department of Physics and Astronomy, University College London, Gower Street, London WC1E 6BT, UK \\ ${ }^{2}$ Department of Physics, Institute for Particle Physics Phenomenology, Durham University, Durham DH1 3LE, UK
}

Received: 11 August 2020 / Accepted: 28 December 2020 / Published online: 19 January 2021

(C) The Author(s) 2021

\begin{abstract}
We scrutinise the ability of the primary QED final-state resummation tools, combined with electroweak virtual corrections, to reproduce the exact next-to-leading order electroweak calculation in the four-charged-lepton final state. We further examine the dependence of the findings on the lepton-photon dressing-cone size as well as the resonance identification strategy. Overall we find excellent agreement with the fixed-order result, but partial differences not directly connected with resummation-induced higher-order effects at the few-percent level are observed in some cases, which are relevant for precision measurements.
\end{abstract}

\section{Introduction}

The production of four charged leptons in proton-proton collisions offers a rich gamut of processes contributing to the same final state, bound through higher-order electroweak effects, in an experimentally clean environment. Precise measurements of this diverse spectrum are crucial for our understanding of irreducible backgrounds in Higgs boson production as well as vector boson scattering topologies, where charge-parity-violating effects could reveal compelling signs of physics beyond the Standard Model [1]. As such, a detailed study of the four-lepton invariant mass, the azimuthal decorrelation and other similar observables in $p p \rightarrow \ell \ell \ell^{\prime} \ell^{\prime}$ production constitutes a vital probe of the gauge structure of the Standard Model whilst providing the ideal test bed to validate state-of-the-art theoretical calculations that feed into the experimental analyses. Both ATLAS and CMS and have produced fiducial differential cross-section measurements of four-lepton production in an inclusive phase space [2] as well as on-shell regions consistent with $Z Z \rightarrow 4 \ell$ production [3,4] and $H \rightarrow Z Z^{*} \rightarrow 4 \ell$ production [5,6]. Differential cross-section measurements of the four-lepton final state

\footnotetext{
a e-mail: c.gutschow@ucl.ac.uk

b e-mail: marek.schoenherr@durham.ac.uk (corresponding author)
}

have already been used to set limits on both charge-parity violation [7] as well as the Higgs self-couplings [8].

Of course, precision measurements necessitate precise calculations to be able to extract as much information as possible. To this end, the next-to-leading order (NLO) QCD corrections to on-shell $Z Z$ production are known for almost three decades $[9,10]$. The off-shell four-lepton production then followed no ten years later $[11,12]$. Recently, the nextto-next-to-leading order (NNLO) QCD corrections were added [13-15], stabilising the cross section predictions on the percent level with respect to the usual QCD scale uncertainties. Although gluon-initiated four lepton production, being a loop-induced process, formally contributes only at NNLO QCD and beyond, its contribution is phenomenologically relevant. Therefore, it was calculated early on [16-19], and even the NLO QCD corrections are known by now [20-22]. In terms of experimentally usable particle-level predictions, at the moment only the NLO QCD calculations are matched to parton showers in various schemes [23-28], benefiting also from the respective event generators' higher-order QED corrections which is especially important for observables sensitive to energy loss through photon radiation.

The electroweak (EW) correction to four-lepton production, on the other hand, were first calculated in the EW Sudakov approximation [29-34], tailored to describe observables sensitive to momentum transfers much larger than the electroweak scale. Photonic corrections, which are of particular importance to observables that contain resonance peaks or thresholds, were analytically calculated in [35]. The complete NLO EW corrections were only calculated in the last ten years [36-39] and were found to be important ingredients in precision phenomenology in four lepton final states. They have recently also been combined with the NNLO QCD corrections to form the highest-precision fixed-order calculation available [40]. During the completion of the present paper, also a first calculation matching the combined NLO QCD and NLO EW corrections to the parton shower has been presented in [41]. 
In the Monte-Carlo event generators currently used by the LHC experiments, NLO QCD matrix elements are matched to parton showers, possibly merging in higher-multiplicity process [42]. Therein, QED corrections are provided by universal QED parton showers [43-47] or other QED-specific resummations [48-51]. Process-specific EW corrections are either applied a posteriori on the level of measured observables by extracting correction factors from the fixed-order calculations or they are applied in either the Sudakov [52,53] or the recently formulated EW virtual approximation [54] on an event-by-event basis.

Therefore, the aim of this paper is to quantify in a tuned comparison the inherent differences of the two commonly used tools for higher-order QED corrections, PHOTOS [48] and SHERPA's Yennie-Frautschi-Suura (YFS) [55] based softphoton resummation [51], combined with the EW virtual approximation, in order to ascertain their ability to reproduce the exact NLO EW results and to be able to quantify the algorithmic uncertainties associated with these corrections. This paper is thus organised as follows: in Sect. 2 we summarise the calculational methods and tools that are used in this paper. In Sect. 3 we then present a detailed comparison and analysis of the quality of the different approximations compared to the fixed-order NLO EW calculation. Finally, we offer our conclusions in Sect. 4.

\section{Computational methods}

In this paper, we compare the results obtained combining a calculation of LO accuracy in the electroweak sector with both a dedicated QED final-state photon radiation resummation and approximate virtual EW corrections in the scheme of [54], for the production of four charged leptons to the exact NLO EW result.

The exact fixed-order NLO EW results have been obtained with the SHERPA+OPENLOOPS [47,56-58] framework, allowing for a fully automated calculation of cross sections and observables at next-to-leading order in the electroweak coupling. In this framework, renormalised virtual amplitudes are provided by OPENLOOPS [57,58], which uses the COLLIER tensor reduction library [59] as well as CUTTOOLS [60] together with the ONELOOP library [61]. All remaining tasks, i.e. the bookkeeping of partonic subprocesses, phase-space integration, and the subtraction of all QED infrared singularities, are provided by SHERPA using the AMEGIC matrix element generator [62-64]. SHERPA in combination with OPENLOOPS (and other providers of renormalised one-loop corrections) has been employed successfully in a range of different calculations $[54,65-74]$ and has been validated against other tools in [75].

The NLO EW corrections to $\mathrm{pp} \rightarrow 4 \ell$ are dominated by either EW Sudakov logarithms of virtual origin or QED loga- rithms stemming from photon radiation off leptons, depending on the kinematic regime. While EW Sudakov logarithms dominate the large $p_{\mathrm{T}}$ or large invariant mass regions, radiative energy loss through photon emission dominates invariant mass distributions below the $Z$-pair threshold or around the resonant $Z$ Breit-Wigner peak in two- and four-lepton invariant masses. This observation allows to construct a simple yet effective high-precision stand-in for a full next-to-leading order matched event generator combining:

(i) The virtual EW approximation. In [54] it was shown that, for observables that are sufficiently inclusive with respect to photon radiation and where all kinematic invariants are large with respect to the electroweak scale, the full NLO EW results can be reproduced with good accuracy by an approximation consisting only of the exact virtual EW corrections, whose infrared divergences have been suitably subtracted. Thus, this approximation, is defined through

$$
\begin{aligned}
\mathrm{d} \sigma_{\text {NLO EW }} & =\mathrm{d} \sigma_{\mathrm{LP} r o x}+\mathrm{d} \sigma_{\mathrm{EW}}^{\mathrm{V}}+\mathrm{d} \sigma_{\mathrm{EW}, \text { approx }}^{\mathrm{R}} \\
& =\mathrm{d} \sigma_{\mathrm{LO}}\left(1+\delta_{\mathrm{EW}_{\text {approx }}}\right) .
\end{aligned}
$$

Therein, $\mathrm{d} \sigma_{\mathrm{LO}}$ is the leading order differential cross section, while $\mathrm{d} \sigma_{\mathrm{EW}}^{\mathrm{V}}$ and $\mathrm{d} \sigma_{\mathrm{EW}}^{\mathrm{R}}$,approx are the exact NLO EW virtual correction and the endpoint part of the emitted-photon-integrated approximate real emission amplitude. ${ }^{1}$ Hence, by construction, $\mathrm{d} \sigma_{\mathrm{EW} \text {,approx }}^{\mathrm{R}}$ does not only ensure a finite result but also supplies real emission QED logarithms to the approximation. This approach captures all Sudakov effects at NLO EW and is also very suitable for a combination of QCD and EW higher-order effects through a simplified multi-jet merging approach at NLO QCD+EW [54,71,74].

(ii) QED final state radiation. The inherent approximation of the above virtual EW approximation is partially unfolded again by employing dedicated final-state photon emission resummations. Specifically, we consider a soft-photon resummation in the Yennie-FrautschiSuura (YFS) scheme [55] as implemented in SHERPA [51] and, alternatively, PHOTOS $[48,76-78] .^{2}$ Both are limited to final state radiation (FSR) and $1 \rightarrow n$ processes, but are currently the tools of choice to calculate QED FSR corrections for the LHC experiments. To understand their FSR resummation properties we

\footnotetext{
1 In practice, the Catani-Seymour I-operator is used.

2 We use the native implementation of the soft-photon resummation of SHERPA 2.2.8, and use the C++ interface to PHOTOS 3.6.4 to directly call PHOTOS from within SHERPA. Both tools are handed the exact same reconstructed $1 \rightarrow n$ subprocesses. Each interface and parameter setup is independent of the process (or reconstructed resonant subprocess) under consideration.
} 
sketch here their defining approximation of the allorders decay rate $\mathrm{d} \Gamma$ in terms of a given LO decay rate $\mathrm{d} \Gamma_{0}$. РнотоS calculates it as

$$
\begin{aligned}
& \mathrm{d} \Gamma^{P \text { HOTOS }} \\
&=\mathrm{d} \Gamma_{0}\left\{1+\sum_{c=1}^{n_{\mathrm{ch}}} \sum_{n_{\gamma}} \frac{\left(\alpha L_{c}\right)^{n_{\gamma}}}{n_{\gamma} !}\left[\prod_{i=1}^{n_{\gamma}} \mathrm{d} x_{c}^{i}\right]\right. \\
&\left.\quad \times\left(P_{\epsilon_{\mathrm{cut}}}\left(x_{c}^{1}\right) \otimes \ldots \otimes P_{\epsilon_{\mathrm{cut}}}\left(x_{c}^{n_{\gamma}}\right)\right)\right\}
\end{aligned}
$$

where the radiative part is summed over all $n_{\mathrm{ch}}$ charged particles. $L_{c}$ is the logarithm of the ratio of the decaying particle's mass over the mass of the charged particle $c$, and $x_{c}=\prod x_{c}^{i}$ is its retained energy fraction after the radiation of $n_{\gamma}$ photons. The phase space distribution of these photons is described by the Altarelli-Parisi splitting functions $P_{\epsilon_{\text {cut }}}(x)$ in the presence of the infrared cut-off $\epsilon_{\text {cut }}$, modified by suitable weights to recover the correct soft-photon limit and implement exact higherorder corrections, and iterated over all $n_{\gamma}$ emitted photons. Their precise definitions can be found in [76]. The implementation of the YFS soft-photon resummation in SHERPA, on the other hand, calculates the all-orders resummed decay rate using

$$
\begin{aligned}
& \mathrm{d} \Gamma^{\mathrm{YFS}}=\mathrm{d} \Gamma_{0} \cdot e^{\alpha Y\left(\omega_{\mathrm{cut}}\right)} \\
& \quad \times \sum_{n_{\gamma}} \frac{1}{n_{\gamma} !}\left[\prod_{i=1}^{n_{\gamma}} \mathrm{d} \Phi_{k_{i}} \cdot \alpha \tilde{S}\left(k_{i}\right) \Theta\left(k_{i}^{0}-\omega_{\mathrm{cut}}\right) \cdot \mathcal{C}\right] .
\end{aligned}
$$

Here, $Y\left(\omega_{\text {cut }}\right)$ is the YFS form factor resumming unresolved real and virtual soft-photon corrections. The individual resolved photon $k_{i}$ 's phase space, $\Phi_{k_{i}}$, is distributed according to the eikonal $\tilde{S}\left(k_{i}\right)$, which is built up by the coherent sum of dipoles formed by all pairs of charged particles in the decay. $\omega_{\text {cut }}$ separates the explicitly-generated resolved from the integrated-over unresolved real photon emission phase space. The correction factor $\mathcal{C}$ restores the correct spin-dependent collinear limit and contains decay-specific exact higherorder correction, cf. [51] for details.

With Eqs. (2.2) and (2.3) at hand, we observe that through the inclusion of exact NLO QED matrix element corrections ${ }^{3}$ to their initial photon distributions (collinear splitting functions in PHOTOS, soft eikonal in

\footnotetext{
3 While NNLO QED + NLO EW corrections are available for the YFS implementation in SHERPA [79] it is currently not the default in the experiments, and thus not employed here.
}

YFS), both resummations should produce very similar results in $Z \rightarrow \ell^{+} \ell^{-}$decays. As both approaches, however, resum different quantities, the logarithm $L_{c}$ in PHOTOS and the YFS form factor $Y$ in the soft-photon resummation, differences are expected when resummation effects become important.

Finally, conversions of photons into lepton pairs is not accounted for in either program. It needs to be noted that both resummations are unitary and do not alter the event weight.

Consequently, the combination of either QED FSR resummation with the virtual EW approximation are dubbed NLO $\mathrm{EW}_{\text {approx }} \times Y$ FS and NLO $\mathrm{EW}_{\text {approx }} \times$ PHOTOS approximations in the following. Its validity was further tested for other classes of processes, among them the production of $2 \ell 2 v$ final states, [66,74]. While this construction is of course not formally NLO accurate, it provides an accurate description of both logarithmically enhanced regions. Its performance will be assessed in detail in Sect. 3 . One crucial input, however, is the treatment of resonances in the QED FSR tools. It is described in the following.

Resonance identification The implementation of resummed final state photon emission corrections in SHERPA includes a generic resonance identification, ensuring that collective multipole radiation off the charged-lepton ensemble preserves all resonance structures present in the event. This is more relevant in soft-photon resummations than in collinear ones, since soft wide-angle emissions have a stronger effect on the lepton direction than collinear ones and are not recombined into a physical dressed lepton momentum. To this end, first the final state of a scattering process is analysed and possible resonances decaying into lepton pairs are identified on the basis of event kinematics and existing vertices in the model. For the process studied in this paper, $\mathrm{pp} \rightarrow \ell^{+} \ell^{-} \ell^{\prime+} \ell^{\prime-}\left(\ell, \ell^{\prime} \in e, \mu\right)$, multiple resonance structures are possible. They are disentangled on the basis of the distance measure $\Delta_{\ell \ell}^{Z}=\left|m_{\ell^{+} \ell^{-}}-m_{Z}\right| / \Gamma_{Z}$, where of course only same-flavour pairs are taken into account. A lepton pair is then considered to be produced by a resonance if $\Delta_{\ell \ell}^{Z}<\Delta_{\text {thr }}$, with $\Delta_{\text {thr }}$ being a free parameter of order 1 . Subsequently, identified resonant-production subprocesses are separated from the rest of the event, and the emerging decay is dressed with photon radiation respecting the Breit-Wigner distribution of the resonance, i.e. preserving the original virtuality of the off-shell leptonic system. Finally, all left-over non-resonantly produced leptons are grouped in a fictitious process, $X \rightarrow \ell^{+} \ell^{-}$or $X \rightarrow \ell^{+} \ell^{-} \ell^{\prime+} \ell^{\prime-}$, with suitably adjusted masses for $X$.

Thus, depending on the four-lepton kinematics, three cases can be distinguished, cf. Fig. 1: 


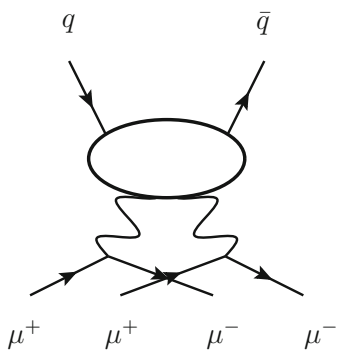

(a)

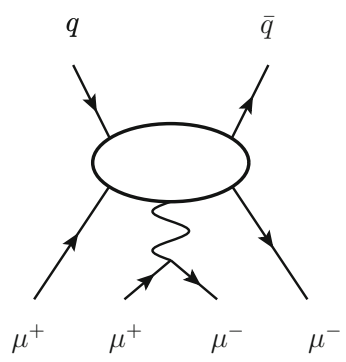

(b)

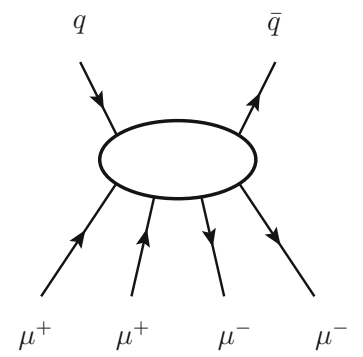

(c)

Fig. 1 Possible resonance structure: a double resonant, b single resonant, and $\mathbf{c}$ non-resonant

(a) Double resonant. Two pairs of opposite sign and same flavour leptons whose respective $\Delta_{\ell \ell}^{Z}$ is smaller than $\Delta_{\text {thr }}$ are identified by the above algorithm. Hence, both $Z \rightarrow \ell^{+} \ell^{-}$decays are reconstructed (setting the $Z$ mass equal to $m_{\ell \ell}$ ) and passed separately to the QED FSR resummation.

(b) Single resonant. Only one pair of opposite sign and same flavour leptons with $\Delta_{\ell \ell}^{Z}$ is smaller than $\Delta_{\text {thr }}$ is found. Only for this pair a $Z \rightarrow \ell^{+} \ell^{-}$decay is reconstructed, and passed on as such to the QED FSR resummation. The remaining leptons are treated as non-resonantly produced and passed to the QED FSR resummation as such. In consequence, no specific $Z \rightarrow \ell^{+} \ell^{-}$higher-order corrections are applied.

(c) Non-resonant. No opposite sign and same flavour lepton pair with $\Delta_{\ell \ell}^{Z}<\Delta_{\text {thr }}$ is found. Consequently, the complete four lepton final state is passed to the QED FSR resummation as is and no specific $Z \rightarrow \ell^{+} \ell^{-}$higherorder corrections are applied.

In essence, due to the inclusivity of the cuts employed for the analysis in Sect.3, the bulk of the cross section is classified as doubly resonant. The precise fraction, however, depends on the free parameter $\Delta_{\mathrm{thr}}$, or the answer to the question when is a lepton pair considered to be produced resonantly or not.

\section{Results}

For the numerical results presented in this section we use the tools and methods summarised in Sect. 2. Both the NLO EW calculation as well as the approximate $\mathrm{NLO} \mathrm{EW}_{\text {approx }} \times Y \mathrm{FS}$ and NLO $\mathrm{EW}_{\text {approx }} \times P$ HOTOS are calculated (and renormalised) in the $G_{\mu}$-scheme with the following input parameters

$$
\begin{aligned}
G_{\mu} & =1.1663787 \times 10^{-5} \mathrm{GeV}^{-2} \\
m_{W} & =80.385 \mathrm{GeV} \\
m_{Z} & =91.1876 \mathrm{GeV} \\
m_{h} & =125.0 \mathrm{GeV} \\
m_{t} & =173.2 \mathrm{GeV}
\end{aligned}
$$

All other particles are considered massless. The electromagnetic coupling is thus defined as

$\alpha_{G_{\mu}}=\left|\frac{\sqrt{2} G_{\mu} \mu_{W}^{2} \sin ^{2} \theta_{w}}{\pi}\right|$,

with the complex masses and mixing angles,

$$
\mu_{i}^{2}=m_{i}^{2}-\mathrm{i} m_{i} \Gamma_{i} \quad \text { and } \quad \sin ^{2} \theta_{w}=1-\frac{\mu_{W}^{2}}{\mu_{Z}^{2}} .
$$

The additional power of $\alpha$ occuring at NLO is set to its value in the Thomson limit,

$\alpha(0)=1 / 137.03599976$

in order to facilitate the comparison to the FSR resummation tools. Higher-order EW corrections are estimated by changing the renormalisation scheme to the $\alpha\left(m_{Z}\right)$ scheme, ${ }^{4}$ still keeping the additional power in the EW coupling at NLO at $\alpha(0)$. As this delivers only a discrete two-point variation, an estimate of the renormalisation scheme uncertainty would be obtained by symmetrising the difference between the two predictions around our chosen central value.

Furthermore, we use the NNPDF30_nnlo_as_0118 PDFs [80], SHERPA's default PDF also used by the LHC experiments, interfaced through LHAPDF 6.2.1 [81]. This choice removes $\gamma$-induced contributions, which both facilitates the comparisons against the QED final-state resummations and has been found to be phenomenologically unimportant $[38,39]$. It also makes our findings directly transferable to current LHC applications which all use this PDF set. However, as we nonetheless include QED initial-state mass factorisation terms to render the NLO EW calculation finite [63], we incur a slight mismatch in the initial-state evolution between the PDF and NLO EW calculation, which again does not impact the comparison presented in the following.

Our results are independent of the QCD renormalisation scale $\mu_{R}$ throughout, and only weakly depend on the factorisation scale $\mu_{F}$. To avoid having to resolve ambiguities in

\footnotetext{
$\overline{4}$ The $\alpha\left(m_{Z}\right)$ scheme is defined by the $W$ and $Z$ masses and widths detailed above in addition to $\alpha\left(m_{Z}\right)=1 / 128.802$.
} 
the same-flavour channel, we simply set it to

$\mu_{F}=\frac{1}{2} \sum_{i=1}^{4} p_{\mathrm{T}, \ell_{i}}$,

where the sum includes all four dressed lepton momenta defined below. In addition, both the YFS soft-photon resummation and Рнотоs use the electromagnetic coupling in the Thomson limit, cf. Eq. (3.3). As infrared cut-offs we use $\omega_{\text {cut }}=1 \mathrm{MeV}$ for the YFS soft-photon resummation, applied to the photon energy in the rest-frame of the radiating multipole after radiation, and $\epsilon_{\mathrm{cut}}=1 \times 10^{-5}$ for PHOTOS, which translates into $\omega_{\text {cut }}=\epsilon_{\text {cut }} \cdot m$ where $m$ is the invariant mass of the reconstructed decaying particle in its rest frame, as detailed in Sect.2. In both cases, we investigate the impact of a conservative and a relaxed choice of clustering threshold, setting $\Delta_{\mathrm{thr}}=1$ and $\Delta_{\mathrm{thr}}=10$ respectively.

We analyse the events with RIVET [82] using an event selection based on a recent ATLAS measurement of the inclusive four-lepton lineshape at $13 \mathrm{TeV}$ [2]. Electrons and muons are defined at the dressed level, meaning the lepton four-momentum is combined with the four-momenta of nearby prompt photons for different dressing-cone sizes. The dressing-cone size itself is varied between $\Delta R_{\text {dress }}=$ $0.005,0.02,0.1,0.2 .^{5}$ Prompt photons used in the dressing procedure are subsequently removed from the final state. Exactly four muons are selected in the same-flavour case or exactly two electrons and two muons in the different-flavour case. All leptons are required to be within a pseudorapidity of $\left|\eta_{\ell}\right|<2.47$ and to have a minimum transverse momentum of $20 \mathrm{GeV}$ for the leading lepton, $15 \mathrm{GeV}$ for the subleading lepton, and $10 \mathrm{GeV}$ and $7 \mathrm{GeV}$ for the third and fourth lepton, respectively. All same-flavour lepton pairs have to be separated by at least $\Delta R=\sqrt{(\Delta \eta)^{2}+(\Delta \phi)^{2}}>0.1$, while a stricter separation of $\Delta R>0.2$ is required for differentflavour leptons. In case the dressing cone size is larger than half of the pairwise lepton separation, photons are combined with the closest lepton.

Exactly two opposite-charge dilepton pairs are required in the event, where the leading lepton pair is chosen to be the one whose invariant dilepton mass is closest to the $Z$-boson resonance. A dilepton invariant mass window of $50 \mathrm{GeV}<$ $m_{\ell \ell}<106 \mathrm{GeV}$ is used for the leading lepton pair, while a dynamic invariant mass cut is employed for the subleading lepton pair, depending on the overall four-lepton invariant mass, $m_{4 \ell}$ using the following sliding-window algorithm:

- for $m_{4 \ell}<100 \mathrm{GeV}$, require $m_{\ell \ell}>5 \mathrm{GeV}$ for the subleading pair;

\footnotetext{
5 We have studied all of the following dressing cone sizes $\Delta R_{\text {dress }}=$ $0.001,0.002,0.005,0.01,0.02,0.05,0.1,0.2,0.5$. We have chosen the above selection to combine readability with instructiveness, bearing in mind practical relevance.
}

- for $100 \mathrm{GeV} \leq m_{4 \ell}<110 \mathrm{GeV}$, require $m_{\ell \ell}>5 \mathrm{GeV}+$ $0.7 \times\left(m_{4 \ell}-100 \mathrm{GeV}\right)$ for the subleading pair;

- for $110 \mathrm{GeV} \leq m_{4 \ell}<140 \mathrm{GeV}$, require $m_{\ell \ell}>12 \mathrm{GeV}$ for the subleading pair;

- for $140 \mathrm{GeV} \leq m_{4 \ell}<190 \mathrm{GeV}$, require $m_{\ell \ell}>5 \mathrm{GeV}+$ $0.76 \times\left(m_{4 \ell}-140 \mathrm{GeV}\right)$ for the subleading pair;

- for $190 \mathrm{GeV} \leq m_{4 \ell}$, require $m_{\ell \ell}>50 \mathrm{GeV}$ for the subleading pair.

This somewhat intricate definition of the fiducial volume increases the number of experimentally cleanly measurable events in particular in the region below the $Z Z$ continuum where at most one of the $Z Z$ bosons can be on-shell. In particular, the $Z \rightarrow 4 \ell$ resonance is strongly enhanced when compared to uniform acceptance criteria for all leptons. For our comparison this has the advantage that the performance of both approximations can be extensively tested in various regimes, each comprising very different resonant structures.

In the following, we compare the Born-level prediction (black) with the exact NLO EW prediction (green) and the

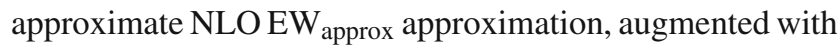
PHOTOS (dotted) or YFS (solid) using either a conservative (red) or relaxed (blue) clustering threshold. We also study the effect of using a range of different dressing-cone sizes, where we expect the dependence of the respective cross sections on the dressing-cone size to be better described by the QED FSR tools than the fixed-order calculations. In particular, we expect both the fixed-order calculations and the QED FSR resummations to agree well for the most inclusive dressingcone size of $\Delta R_{\text {dress }}=0.2$, while the largest dressing-conesize induced deviations are to be expected for the smallest size of $\Delta R_{\text {dress }}=0.005$.

\subsection{Inclusive cross sections}

Before we turn to discuss several classes of differential distribution we briefly scrutinise the inclusive cross section in the fiducial phase space described above. Table 1 summarises these inclusive fiducial cross section for both the sameflavour and different-flavour channel and the representative lepton dressing cone of $\Delta R_{\text {dress }}=0.1$. Most notable, the fixed-order cross section displays a marked dependence on the EW input and renormalisation scheme as it is proportional to $\alpha^{4}$ at the leading order. To estimate the uncertainty due to missing higher-order EW corrections, we vary the renormalisation scheme from our default, the $G_{\mu}$ scheme, to the $\alpha\left(m_{Z}\right)$ scheme. Both schemes are generally considered suitable for the processes under consideration. Indeed, the NLO corrections in the $G_{\mu}$ and $\alpha\left(m_{Z}\right)$ schemes are both at the few-percent level, albeit of opposite sign: $-4.9 \%(-4.8 \%)$ vs. $+2.6 \%(+2.7 \%)$ in the different-flavour (same-flavour) channel, respectively. In any case, in line with our expectation, the EW scheme-uncertainty decreases from $9.8 \%$ at LO 
Table 1 The LO and NLO EW prediction, including their renormalisation scheme uncertainty, for the inclusive fiducial cross sections for a lepton dressing cone size of $\Delta R_{\text {dress }}=0.1$ is compared to predic- tions in the $\mathrm{EW}_{\text {approx }}$ approximation, augmented with PHOTOS or YFS using either a conservative $\left(\Delta_{\mathrm{thr}}=1\right)$ or relaxed $\left(\Delta_{\mathrm{thr}}=10\right)$ clustering threshold

\begin{tabular}{|c|c|c|c|c|c|c|}
\hline \multirow[t]{3}{*}{$e^{+} e^{-} \mu^{+} \mu^{-}$production } & \multicolumn{6}{|c|}{ Inclusive cross-section [fb] } \\
\hline & \multirow[t]{2}{*}{$\overline{\mathrm{LO}}$} & \multirow[t]{2}{*}{ NLO EW } & \multicolumn{2}{|c|}{$\mathrm{NLO} \mathrm{EW}_{\text {approx }} \times Y$ FS } & \multicolumn{2}{|c|}{$\mathrm{NLO} \mathrm{EW}_{\text {approx }} \times P$ HOTOS } \\
\hline & & & $\overline{\Delta_{\mathrm{thr}}}=1$ & $\Delta_{\mathrm{thr}}=10$ & $\overline{\Delta_{\mathrm{thr}}}=1$ & $\Delta_{\mathrm{thr}}=10$ \\
\hline$\alpha_{G_{\mu}}^{4} \cdot \alpha(0)$ scheme & 15.25 & 14.50 & 14.46 & 14.47 & 14.49 & 14.49 \\
\hline$\alpha^{4}\left(m_{Z}\right) \cdot \alpha(0)$ scheme & 13.75 & 14.11 & 14.11 & 14.12 & 14.21 & 14.21 \\
\hline \multirow[t]{3}{*}{$\mu^{+} \mu^{-} \mu^{+} \mu^{-}$production } & \multicolumn{6}{|c|}{ Inclusive cross-section [fb] } \\
\hline & \multirow[t]{2}{*}{$\overline{\mathrm{LO}}$} & \multirow[t]{2}{*}{ NLO EW } & \multicolumn{2}{|c|}{ NLO EW ${ }_{\text {approx }} \times Y$ FS } & \multicolumn{2}{|c|}{ NLO EW approx $\times P$ HOTOS } \\
\hline & & & $\overline{\Delta_{\mathrm{thr}}}=1$ & $\Delta_{\mathrm{thr}}=10$ & $\overline{\Delta_{\mathrm{thr}}}=1$ & $\Delta_{\mathrm{thr}}=10$ \\
\hline$\alpha_{G_{\mu}}^{4} \cdot \alpha(0)$ scheme & 8.99 & 8.56 & 8.54 & 8.54 & 8.55 & 8.55 \\
\hline$\alpha^{4}\left(m_{Z}\right) \cdot \alpha(0)$ scheme & 8.11 & 8.33 & 8.34 & 8.34 & 8.36 & 8.36 \\
\hline
\end{tabular}

to $2.7 \%$ at NLO. It is to be expected though that in regions of phase space with larger EW corrections this uncertainty rises as well. Finally, given this higher-order uncertainty, the NLO EW $\mathrm{Epprox}_{\text {aps }} \times Y$ and NLO $\mathrm{EW}_{\text {approx }} \times P$ HOTOS approximations very well reproduce the exact result to within less than $0.5 \%$. By their construction, including the exact renormalised virtual contributions, they also well reproduce the exact renormalisation scheme dependence. The agreement for the other, somewhat less standard, dressing cones can be gauged from Fig. 6. Disagreements for both stay well below $1 \%$ for $\Delta R_{\text {dress }}=0.2$ and 0.02 , only rising to slightly above $1 \%$ for $\Delta R_{\text {dress }}=0.005$, in line with our earlier expectation. At this point it is again imperative to stress that this excellent level of agreement is to some degree accidental: despite the well-motivated construction of the approximation it is formally not NLO EW accurate. As an example, this level of agreement for inclusive cross sections was not observed in, e.g., $\mu^{+} v_{\mu} e^{-} \bar{v}_{e}$ production [74].

\subsection{Lepton transverse momentum distributions}

The first class of observables we are examining are the transverse momentum distributions of the four leptons. They are shown in Figs. 2, 3, 4 and 5, respectively. Looking at the fixed-order result first, its renormalisation scheme uncertainty increases as the size of the NLO EW correction gets larger, rising from slightly over $2 \%$ in the peak of each distribution to quickly to more than $5 \%$ as the transverse momenta increase.

The dominant effect of the electroweak corrections in the lepton transverse momentum distributions is a depletion of the cross-section in the high $p_{\mathrm{T}}$ tails through the EW Sudakov logarithms, which is well reproduced by the NLO EW approx $\times Y$ FS and NLO EW approx $\times P$ HOTOS approximations in all distributions. Deviations are typically much smaller than the EW renormalisation scheme uncertainty.
When comparing the two approximations to the fixed-order calculation, it can be seen that for both the different-flavour and same-flavour channel both PнОTOS and YFs behave similarly across the spectrum, except for the low- $p_{\mathrm{T}}$ end of the leading and second-leading lepton $p_{\mathrm{T}}$ distribution. Here, depending on the dressing-cone size, YFS slightly undershoots the fixed-order calculation. The effect is most pronounced just below the peak of the respective distribution. This behaviour can be attributed to the fact that the YFS soft-photon resummation has more wide-angle radiation than PHOTOS that will not be recombined into the dressed lepton object. In turn, this causes more events to fail the minimum $p_{\mathrm{T}}$ requirements of both leptons, leading to the correspondingly slightly reduced inclusive cross section already reported in Table 1. A similar effect is not present in the third and fourth lepton in the $p_{\mathrm{T}}$ region under consideration.

While the lepton $p_{\mathrm{T}}$ distributions are generally insensitive to the choice of clustering threshold $\Delta_{\mathrm{thr}}$, a small dependence on the size of the dressing-cone size can be seen, which can be expected since the amount of FSR radiation off the leptons captured by the dressing algorithm determines whether or not the event will pass the fiducial selection. The two larger dressing-cone sizes are more inclusive and so generally better reproduce the fixed-order calculation, which in turn is not expected to reasonably describe the energy profile within the cone. This is where the resummation employed by the two approximations becomes relevant in order to describe the dressing-cone dependence accurately.

\subsection{Four-lepton observables}

Similar to the individual lepton $p_{\mathrm{T}}$ spectra, both PHOTOS and the YFS-based resummation agree well with the fixed-order calculation also for multi-lepton observables in the differentand same-flavour channels. In almost all regions their deviation from the exact result is much smaller than the renor- 

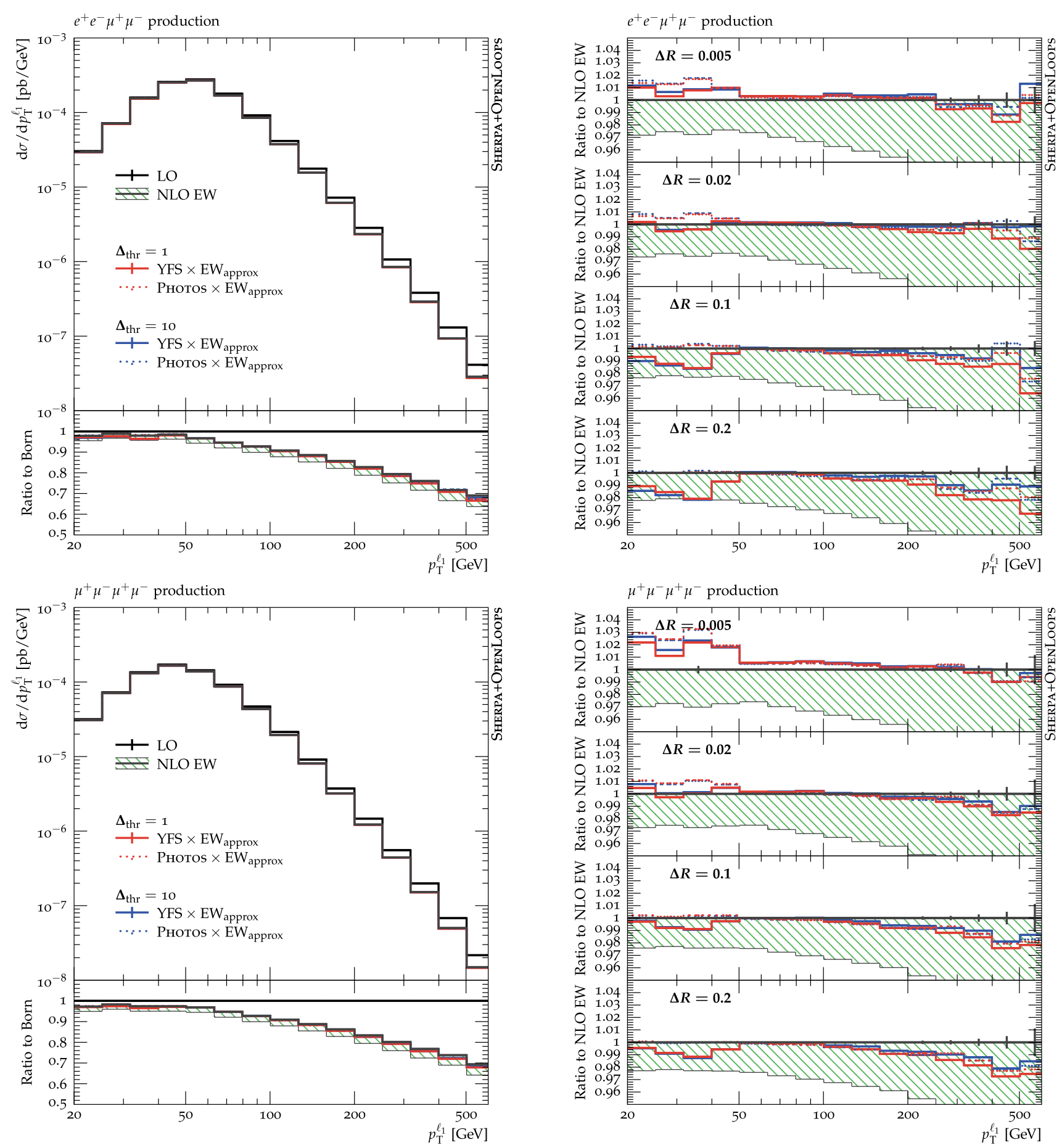

Fig. 2 Differential cross-sections as a function of $p_{\mathrm{T}}^{\ell_{1}}$ in $e^{+} e^{-} \mu^{+} \mu^{-}$ production (top) as well as in $\mu^{+} \mu^{-} \mu^{+} \mu^{-}$production (bottom). The NLO EW prediction (green), including its renormalisation scheme uncertainty, is compared to predictions in the $\mathrm{EW}_{\text {approx }}$ approximation, augmented with PHOTOS (dotted) or YFS (solid) using either a con-

servative (red) or relaxed (blue) clustering threshold. The Born-level prediction is illustrated by the black curve. The absolute cross-sections are shown on the left for a dressing-cone size of 0.1 , while ratios of the РнотоS and YFS curves are shown with respect to the NLO EW prediction on the right for different dressing-cone sizes 

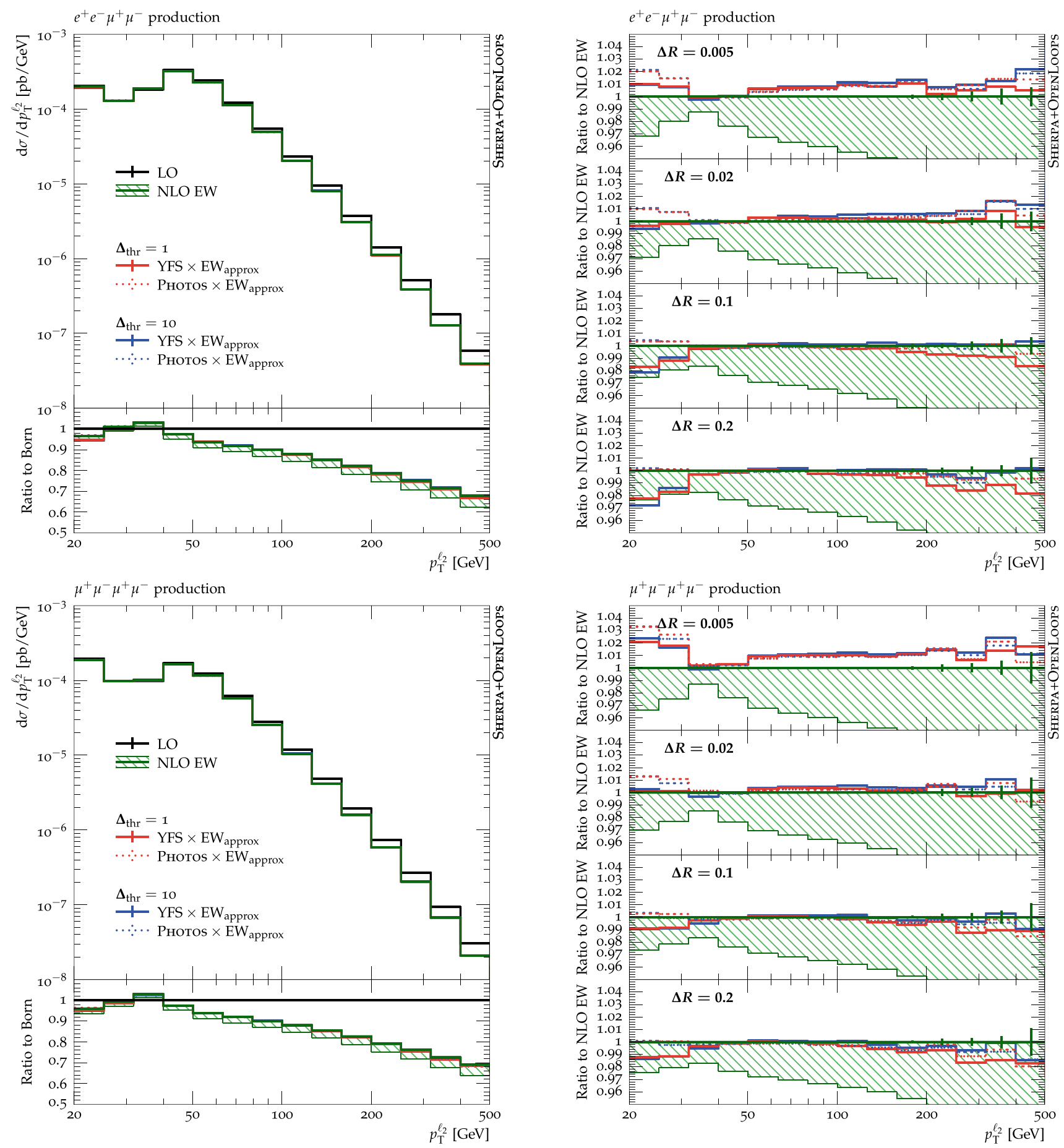

Fig. 3 Differential cross-sections as a function of $p_{\mathrm{T}}^{\ell_{2}}$ in $e^{+} e^{-} \mu^{+} \mu^{-}$ production (top) as well as in $\mu^{+} \mu^{-} \mu^{+} \mu^{-}$production (bottom). The NLO EW prediction (green), including its renormalisation scheme uncertainty, is compared to predictions in the $\mathrm{EW}_{\text {approx }}$ approximation, augmented with PнотоS (dotted) or YFS (solid) using either a con-

servative (red) or relaxed (blue) clustering threshold. The Born-level prediction is illustrated by the black curve. The absolute cross-sections are shown on the left for a dressing-cone size of 0.1 , while ratios of the PнотоS and YFs curves are shown with respect to the NLO EW prediction on the right for different dressing-cone sizes 

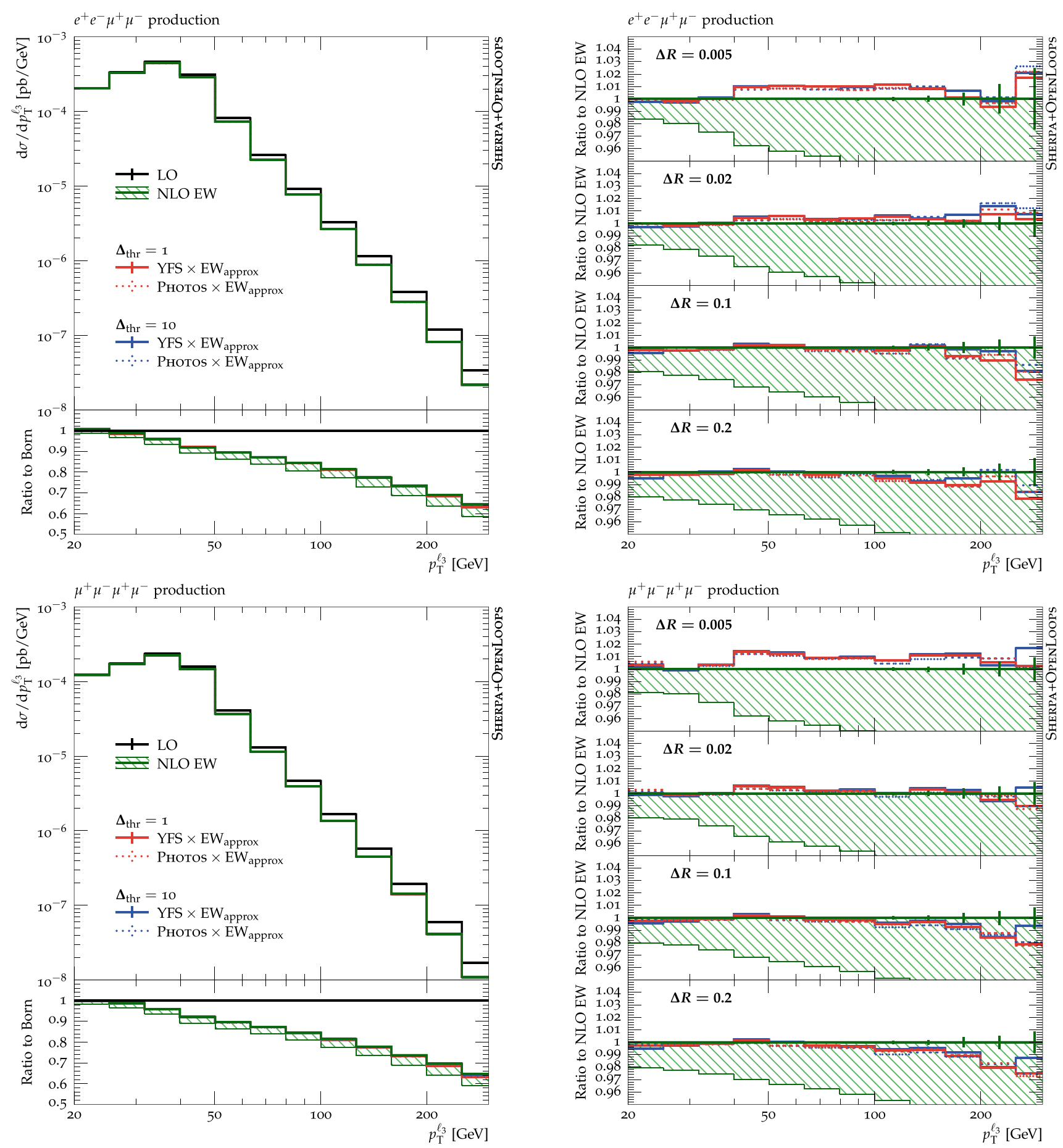

Fig. 4 Differential cross-sections as a function of $p_{\mathrm{T}}^{\ell_{3}}$ in $e^{+} e^{-} \mu^{+} \mu^{-}$ production (top) as well as in $\mu^{+} \mu^{-} \mu^{+} \mu^{-}$production (bottom). The NLO EW prediction (green), including its renormalisation scheme uncertainty, is compared to predictions in the $\mathrm{EW}_{\text {approx }}$ approximation, augmented with PнотоS (dotted) or YFS (solid) using either a con-

servative (red) or relaxed (blue) clustering threshold. The Born-level prediction is illustrated by the black curve. The absolute cross-sections are shown on the left for a dressing-cone size of 0.1 , while ratios of the PнотоS and YFs curves are shown with respect to the NLO EW prediction on the right for different dressing-cone sizes 

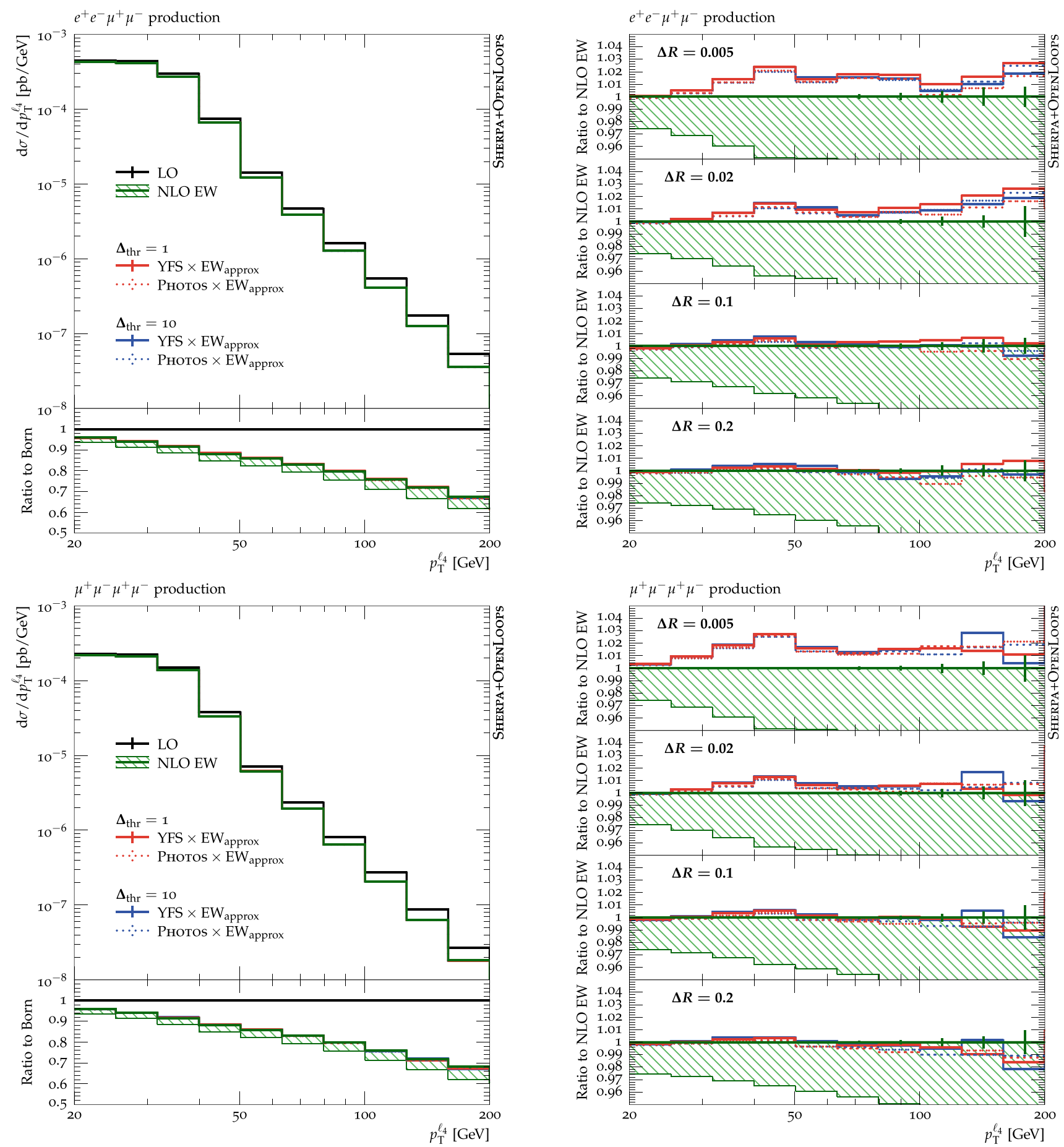

Fig. 5 Differential cross-sections as a function of $p_{\mathrm{T}}^{\ell_{4}}$ in $e^{+} e^{-} \mu^{+} \mu^{-}$ production (top) as well as in $\mu^{+} \mu^{-} \mu^{+} \mu^{-}$production (bottom). The NLO EW prediction (green), including its renormalisation scheme uncertainty, is compared to predictions in the $\mathrm{EW}_{\text {approx }}$ approximation, augmented with PнотоS (dotted) or YFS (solid) using either a con-

servative (red) or relaxed (blue) clustering threshold. The Born-level prediction is illustrated by the black curve. The absolute cross-sections are shown on the left for a dressing-cone size of 0.1 , while ratios of the PнOTOS and YFS curves are shown with respect to the NLO EW prediction on the right for different dressing-cone sizes 
malisation scheme uncertainty, which can be seen in the fourlepton rapidity distribution in Fig. 6 but also in the four-lepton invariant mass spectrum in Fig. 7. As before, the fixed-order scheme uncertainty increases as the overall size of the electroweak correction increases. However, this uncertainty is estimated only by a discrete two-point variation, producing pinch-points whenever the two schemes switch their roles as the one predicting the larger cross section. The thus assessed uncertainty, even after symmetrisation, is underestimated in these regions and should be compared with nearby regions away from the pinch points.

The four-lepton invariant mass distribution covers a wide range of topologies: the $Z Z$ continuum sharply turns on around $180 \mathrm{GeV}$, just before the horizontal axis transitions from a linear to a logarithmic scale at $200 \mathrm{GeV}$. Below the continuum threshold, one of the bosons has to be increasingly off-shell and the cross-section drops accordingly. The cross section then experiences a small rise caused by the virtuality of the off-shell $\gamma^{*}$ to move towards zero until such topologies are disallowed by the otherwise comparably inclusive cuts on the subleading leptons. For $m_{4 \ell} \approx m_{Z}$ the $Z \rightarrow 4 \ell$ peak is well developed, again due to the loose cuts on the subleading leptons which allow for a large number of the preferred hierarchical structure in $Z \rightarrow \ell \ell \gamma[\rightarrow \ell \ell]$ decays. With the leptons of the subleading pair allowed to become soft, a Drell-Yan-like topology is picked out where a primary lepton pair radiates a photon that subsequently splits into a secondary lepton pair with a typically much smaller invariant mass. Since this topology is described with fixed-order matrix elements, all possible combinations and interferences between primary and secondary lepton pair are accounted for.

QED final-state radiation that is not captured by the dressing algorithm will cause the four-lepton system to lose energy and hence migrate from higher to lower invariant mass values. The effect will be largest, with corrections reaching $\mathcal{O}(1)$, just below the $Z$ resonance and the $Z Z$ continuum threshold due events migrating from these regions of enhanced crosssection through radiative energy loss. The precise size of this effect, however, strongly depends on the size of the dressing cone, as it determines how much photon radiation is recombined. These effects are seen in the NLO EW fixed-order prediction and are well reproduced by both approximations for large dressing-cone sizes. As expected, the differences increase the smaller $\Delta R_{\text {dress }}$, with the resummations again being expected to yield more reliable results for very small dressing-cone sizes.

In the off-shell regions below the resonances, the impact of the different clustering thresholds, which determine when a lepton-pair is considered to be produced resonantly, also becomes visible. Not unexpectedly, the effect is larger in the $4 \mu$-channel than in the $2 e 2 \mu$-channel, as the number of potential pairings is larger. Generally, it can be observed that the tighter clustering threshold is somewhat too strict, whereas the looser threshold typically reproduces the full fixed-order calculation better in this region of phase space. Overall, due to its construction around the collinear limit, PHOTOS shows a smaller clustering threshold dependence than the soft-photon resummation of YFS, except for extremely low four-lepton invariant masses.

The large invariant-mass tails are dominated by virtual EW Sudakov logarithms, but a residual dressing-cone-size dependence remains. In all cases, Рнотоs and YFS give almost identical results in both the different-flavour and same-flavour channels. For the most inclusive $\Delta R_{\text {dress }}$ they also excellently agree with the fixed-order calculation, as expected.

\subsection{Lepton-pair observables}

Turning now to lepton-pair observables, Fig. 8 shows the invariant mass of the muon pair in the different-flavour process in the top row and the opposite-sign lepton pair whose invariant is closest to the nominal $Z$ mass for the same-flavour process in the bottom row. In both cases the expected resonance around $91 \mathrm{GeV}$ is accompanied by a smaller enhancement at lower invariant mass values, the shape of which is induced by the fiducial selection criteria. The region below $50 \mathrm{GeV}$ and above $106 \mathrm{GeV}$ is only filled in the differentflavour case where the identification of the two lepton-pairs, and $Z$ candidates, is unambiguous and therefore, the muonpair may be very far off-shell. Whereas in the same-flavour case the leptons, and corresponding $Z$ candidates are identified by choosing the one out of four possible pairings which has the closest invariant mass to the nominal $Z$ mass, and is thus limited by the event selection to a minimal and maximal value of 50 and $106 \mathrm{GeV}$, respectively. The biggest effect of the electroweak corrections is then again seen just below the $Z$ resonance and the selection-induced enhancement below.

Again, there is good agreement between the FSR resummations and the fixed-order calculation for inclusive dressingcone sizes, in particular compared to the fixed-order resummation scheme uncertainty, though as before, differences grow larger for smaller $\Delta R_{\text {dress. }}$ The dependence on the clustering threshold $\Delta_{\text {thr }}$ is also larger for the YFS softphoton resummation than for PHOTOS, with the conservative $\Delta_{\mathrm{thr}}=1$ being too restrictive.

The corresponding transverse momentum spectra are shown in Fig. 9, which also features a cut-induced enhancement around $20-30 \mathrm{GeV}$ as well as the usual electroweak Sudakov suppression in the tail of the distribution. Variations of the dressing-cone size result in a global shift of the two approximations compared to the fixed-order calculation where the latter tends to be better reproduced by the larger dressing-cone sizes. A notable exception here is the aforementioned cut-induced hump around $25 \mathrm{GeV}$ where the 

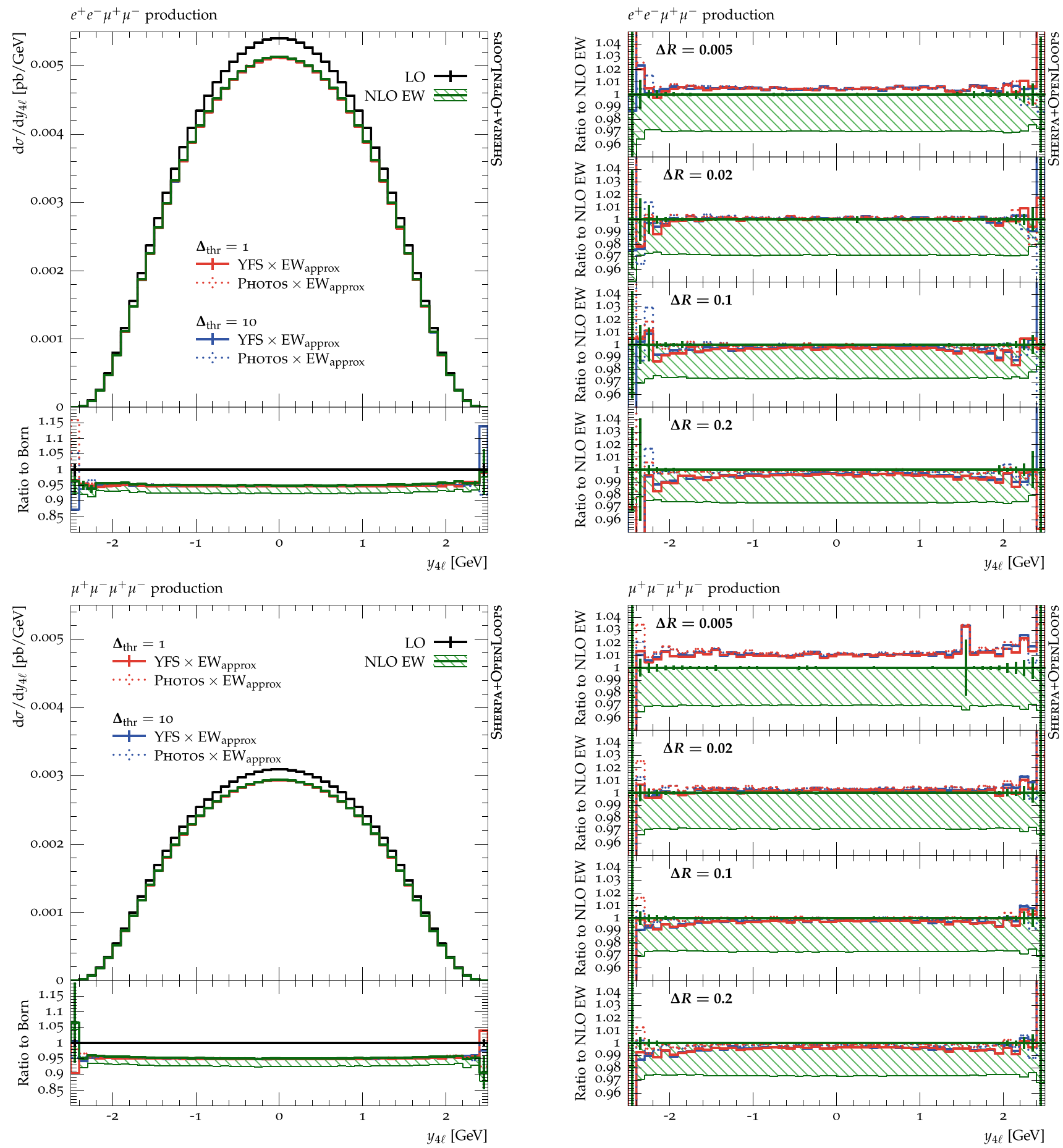

Fig. 6 Differential cross-sections as a function of four-lepton rapidity distribution for $e^{+} e^{-} \mu^{+} \mu^{-}$production (top) as well as $\mu^{+} \mu^{-} \mu^{+} \mu^{-}$ production (bottom). The NLO EW prediction (green), including its renormalisation scheme uncertainty, is compared to predictions in the EW $_{\text {approx }}$ approximation, augmented with PнOTOS (dotted) or YFS (solid) using either a conservative (red) or relaxed (blue) clustering

threshold. The Born-level prediction is illustrated by the black curve. The absolute cross-sections are shown on the left for a dressing-cone size of 0.1 , while ratios of the PHOTOS and YFs curves are shown with respect to the NLO EW prediction on the right for different dressingcone sizes 

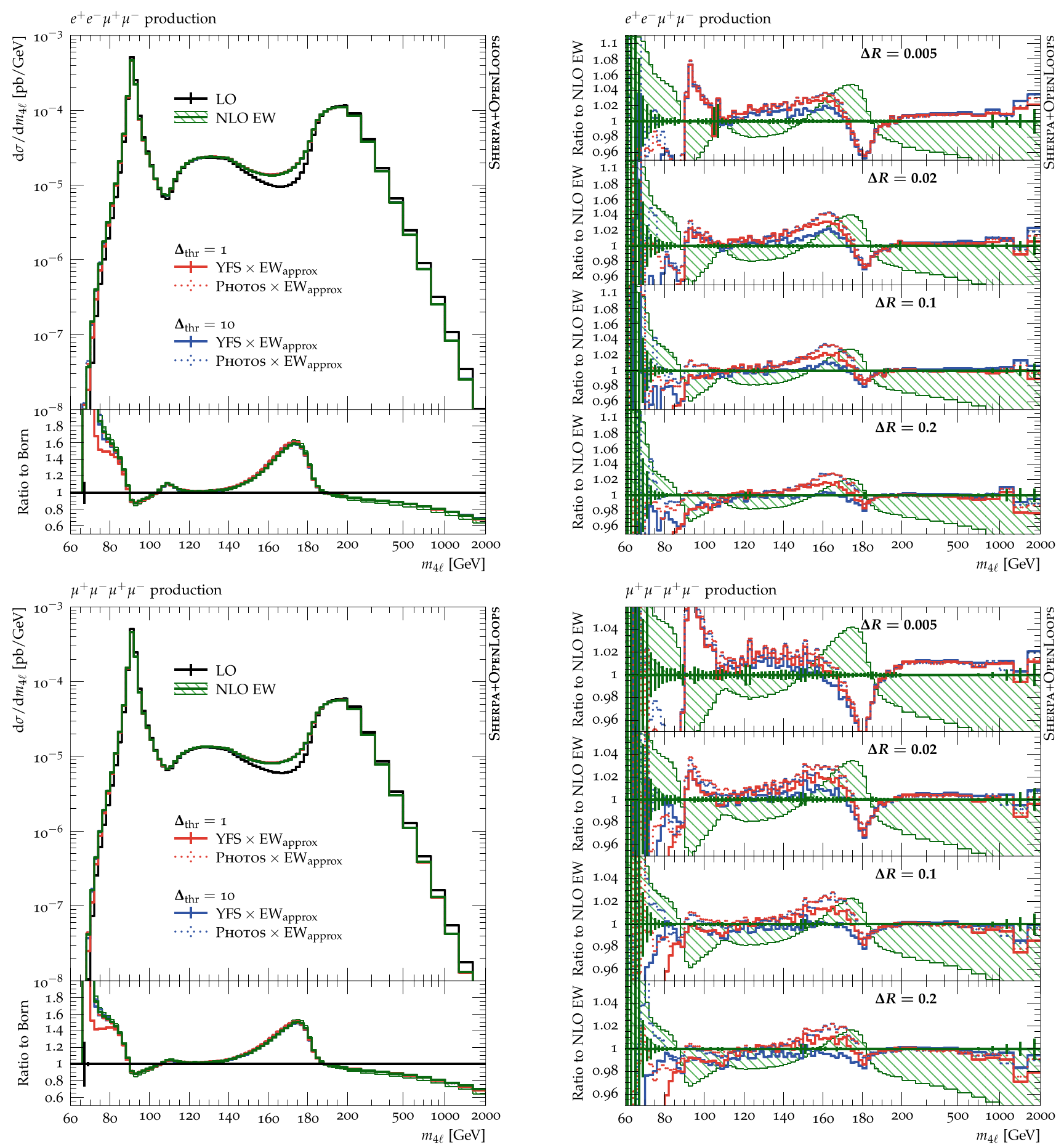

Fig. 7 Differential cross-sections as a function of the four-lepton invariant mass for $e^{+} e^{-} \mu^{+} \mu^{-}$production (top) as well as $\mu^{+} \mu^{-} \mu^{+} \mu^{-}$ production (bottom). The NLO EW prediction (green), including its renormalisation scheme uncertainty, is compared to predictions in the $\mathrm{EW}_{\text {approx }}$ approximation, augmented with PHOTOS (dotted) or YFS (solid) using either a conservative (red) or relaxed (blue) clustering

threshold. The Born-level prediction is illustrated by the black curve. The absolute cross-sections are shown on the left for a dressing-cone size of 0.1 , while ratios of the PHOTOS and YFs curves are shown with respect to the NLO EW prediction on the right for different dressingcone sizes 

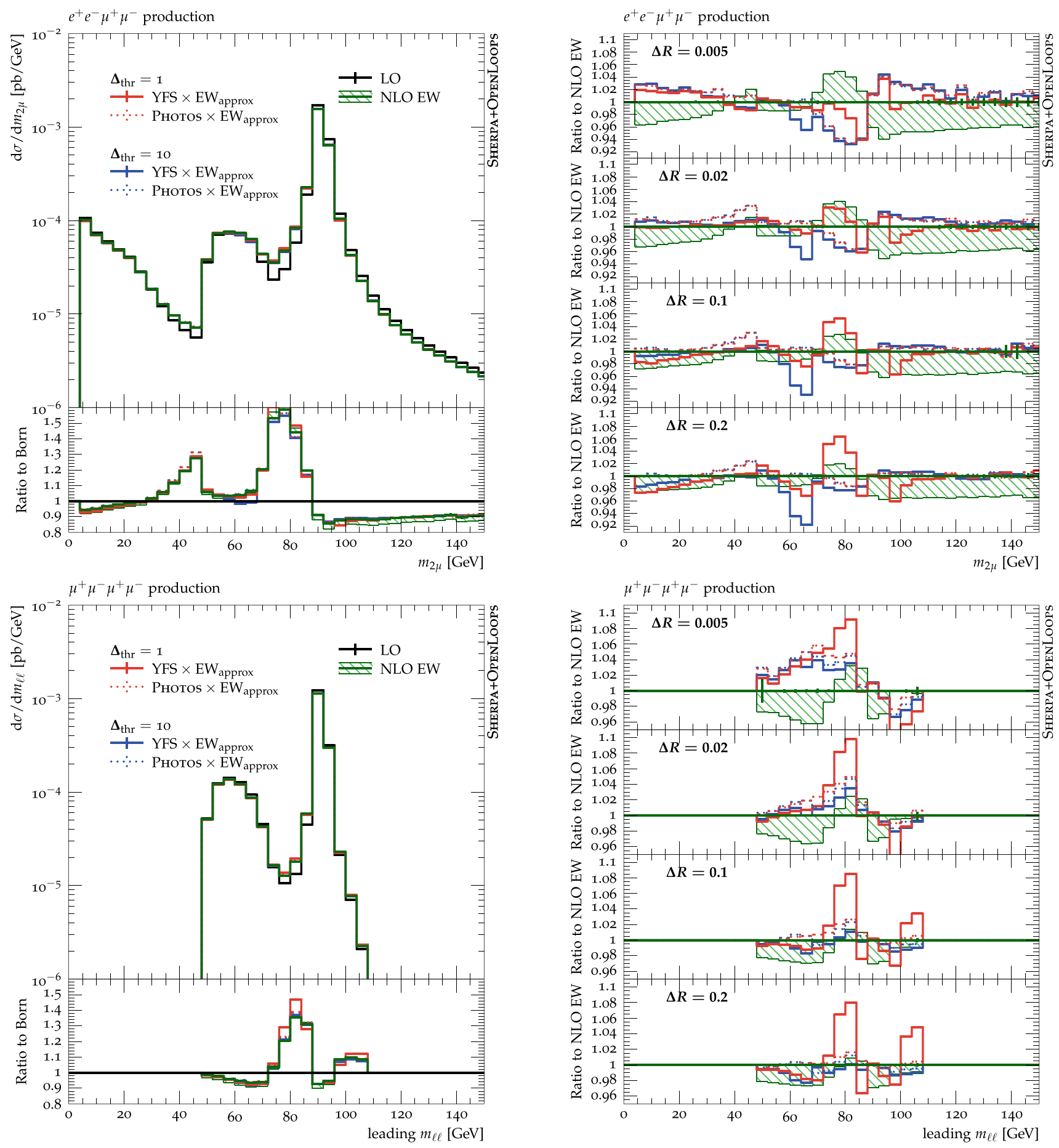

Fig. 8 Differential cross-sections as a function of the invariant mass of the muon pair in $e^{+} e^{-} \mu^{+} \mu^{-}$production (top) as well as the invariant mass of the leading muon pair in $\mu^{+} \mu^{-} \mu^{+} \mu^{-}$production (bottom). The NLO EW prediction (green), including its renormalisation scheme uncertainty, is compared to predictions in the $\mathrm{EW}_{\text {approx }}$ approximation, augmented with PHOTOS (dotted) or YFS (solid) using either a con-

servative (red) or relaxed (blue) clustering threshold. The Born-level prediction is illustrated by the black curve. The absolute cross-sections are shown on the left for a dressing-cone size of 0.1 , while ratios of the PнотOS and YFs curves are shown with respect to the NLO EW prediction on the right for different dressing-cone sizes 

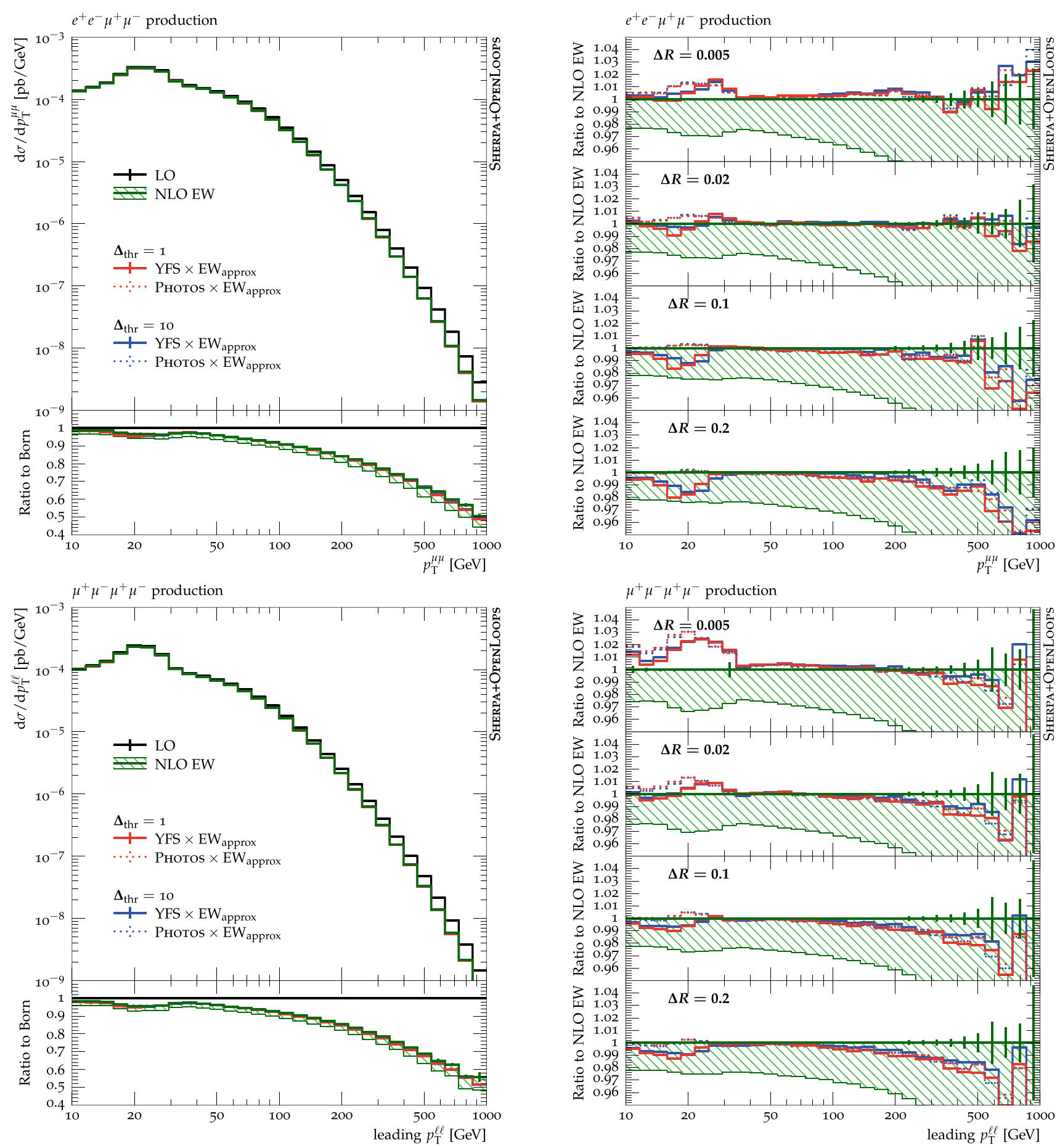

Fig. 9 Differential cross-sections as a function of the transverse momentum of the muon pair in $e^{+} e^{-} \mu^{+} \mu^{-}$production (top) as well as the transverse momentum of the leading muon pair in $\mu^{+} \mu^{-} \mu^{+} \mu^{-}$ production (bottom). The NLO EW prediction (green), including its renormalisation scheme uncertainty, is compared to predictions in the $\mathrm{EW}_{\text {approx }}$ approximation, augmented with PнOTOS (dotted) or YFS

(solid) using either a conservative (red) or relaxed (blue) clustering threshold. The Born-level prediction is illustrated by the black curve. The absolute cross-sections are shown on the left for a dressing-cone size of 0.1 , while ratios of the PнотоS and YFs curves are shown with respect to the NLO EW prediction on the right for different dressingcone sizes 


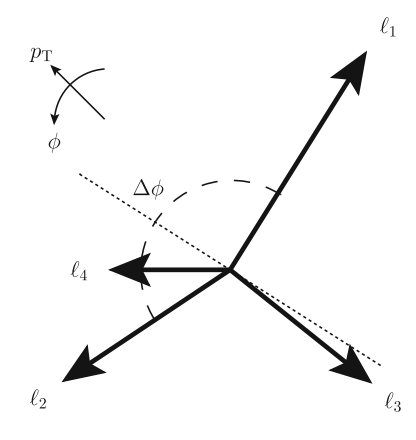

(a)

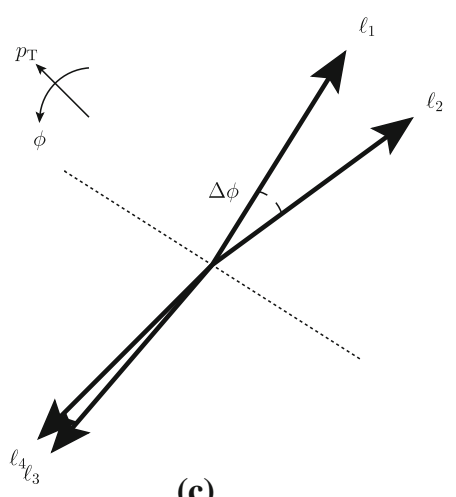

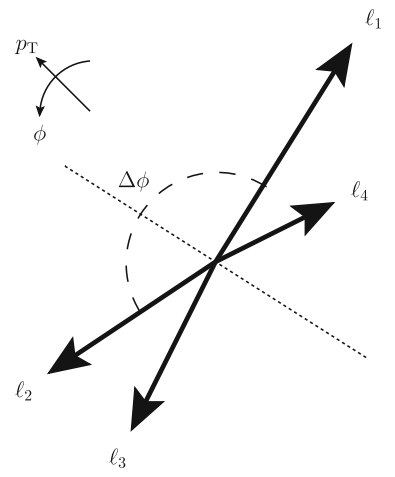

(b)

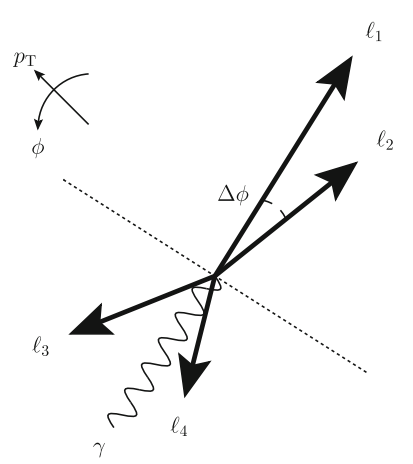

(d)

Fig. 10 Sketch of possible phase space configurations of the four lepton final state in the $p_{\mathrm{T}}-\phi$ plane

EW corrections display a stronger dressing-cone-size dependence. Both effects are not surprising as every cut in the fiducial selection adds sensitivity to the modelling of QED final-state radiation, which is required to accurately describe the fraction of events predicted to pass the selection cuts.

Although the transverse momentum observables display hardly any dependence on $\Delta_{\text {thr }}$, the YFS soft-photon resummation and Рнотоs predict noticeably different results on the $1 \%$ level below $\approx 30 \mathrm{GeV}$, with Рнотоs being consistently larger for every considered dressing cone size in both the same-flavour as well as the different-flavour channel.

\subsection{Azimuthal correlations}

Figure 10 shows a few possible phase-space configurations of the four-lepton final state in the $p_{\mathrm{T}}-\phi$ plane. In the Born configuration, the leading two leptons are typically in opposite hemispheres resulting in a large azimuthal difference between them. Here, either the leading lepton $\ell_{1}$ balances all three subleading leptons $\ell_{2}, \ell_{3}$ and $\ell_{4}$ (a), or either the third or fourth lepton may cross over to the leading lepton's hemisphere (b). In order for the azimuthal opening angle $\Delta \phi$ between the leading and the subleading lepton to become small, and in particular for the subleading lepton to cross over into the leading lepton's hemisphere, both the relative transverse momenta of all four leptons have to become almost degenerate and the opening angle between the third and fourth lepton has to be smaller than that of the leading and subleading one (c). All of these restrictions are lifted once an additional object to recoil against is present (d), greatly enhancing the available phase space for configurations with small $\Delta \phi\left(\ell_{1}, \ell_{2}\right)$.

Figure 11 now displays the azimuthal separation of the two leading leptons, showing exactly the aforementioned suppression for small $\Delta \phi$ at leading order. For $\Delta \phi\left(\ell_{1}, \ell_{2}\right)>\frac{\pi}{2}$, where the leading and subleading leptons reside in opposite hemispheres, the NLO EW corrections and their uncertainties are roughly constant and reproduce the total NLO EW corrections to the inclusive cross section. Here, both YFS and РнотоS agree well with the fixed-order calculation, with deviations in the permille range being much smaller than the renormalisation scheme uncertainty of $2.5-3 \%$, for the most inclusive dressing-cone sizes. The smaller dressing cones again induce shape and rate differences between the resummations and the fixed-order result. Only minute $\Delta_{\text {thr }}{ }^{-}$ dependences can be observed.

In the region $\Delta \phi\left(\ell_{1}, \ell_{2}\right)<\frac{\pi}{2}$ now, the NLO EW corrections, through the presence of the additional real emission 

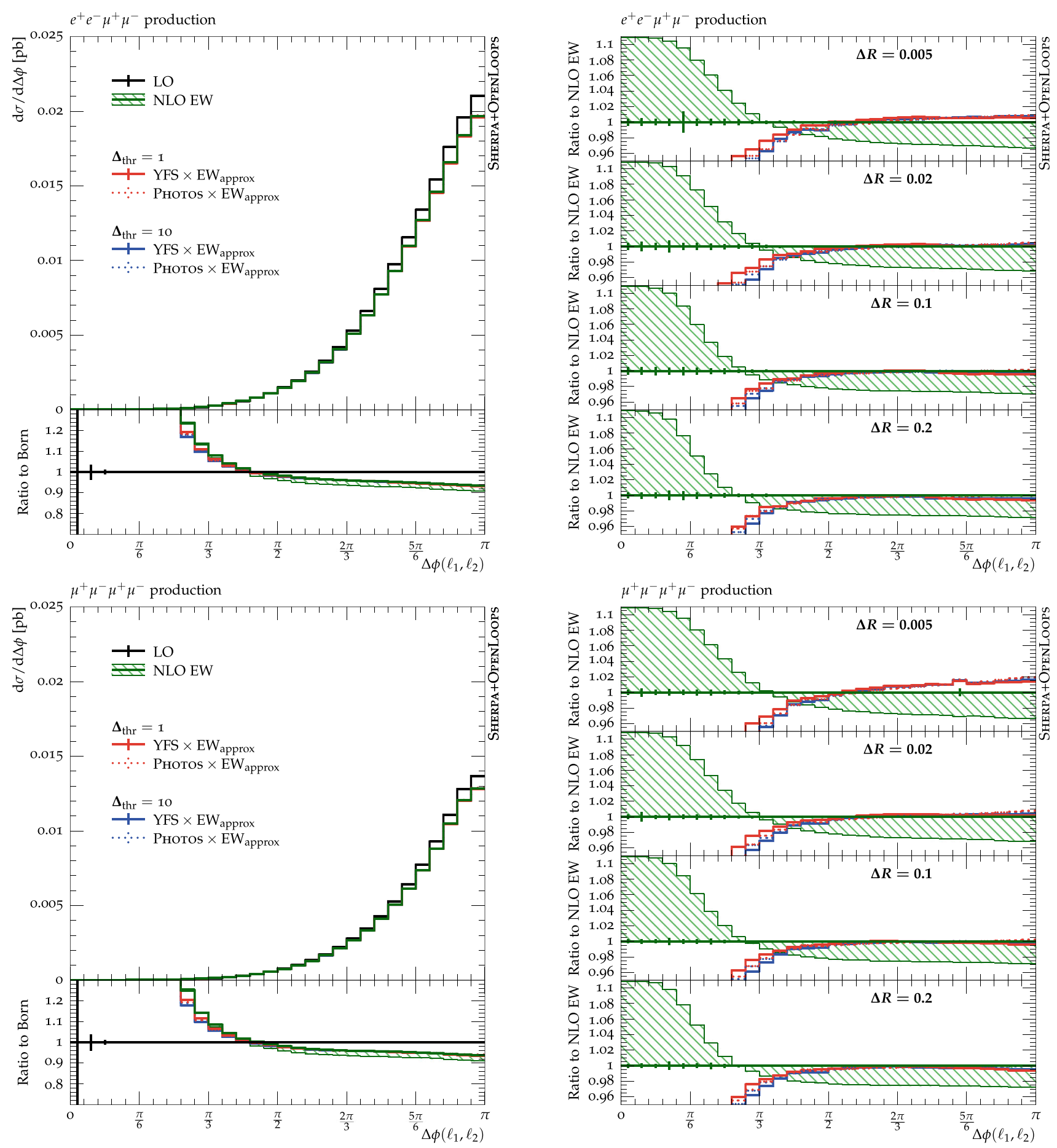

Fig. 11 Differential cross-sections as a function of the azimuthal separation between the leading and subleading lepton, $\Delta \phi\left(\ell_{1}, \ell_{2}\right)$, in $e^{+} e^{-} \mu^{+} \mu^{-}$production (top) as well as $\mu^{+} \mu^{-} \mu^{+} \mu^{-}$production (bottom). The NLO EW prediction (green), including its renormalisation scheme uncertainty, is compared to predictions in the $\mathrm{EW}_{\text {approx }}$ approximation, augmented with PHOTOS (dotted) or YFS (solid) using either a

photon, lifts the above-discussed kinematic restrictions and induce strongly increasing positive EW corrections, although the absolute cross section in this region remains tiny. Corre-

conservative (red) or relaxed (blue) clustering threshold. The Born-level prediction is illustrated by the black curve. The absolute cross-sections are shown on the left for a dressing-cone size of 0.1 , while ratios of the PHOTOS and YFs curves are shown with respect to the NLO EW prediction on the right for different dressing-cone sizes

spondingly, as this correction is driven by the real emission corrections only, the scheme uncertainty becomes leadingorder-like and increases to over $10 \%$. Nonetheless, as the 

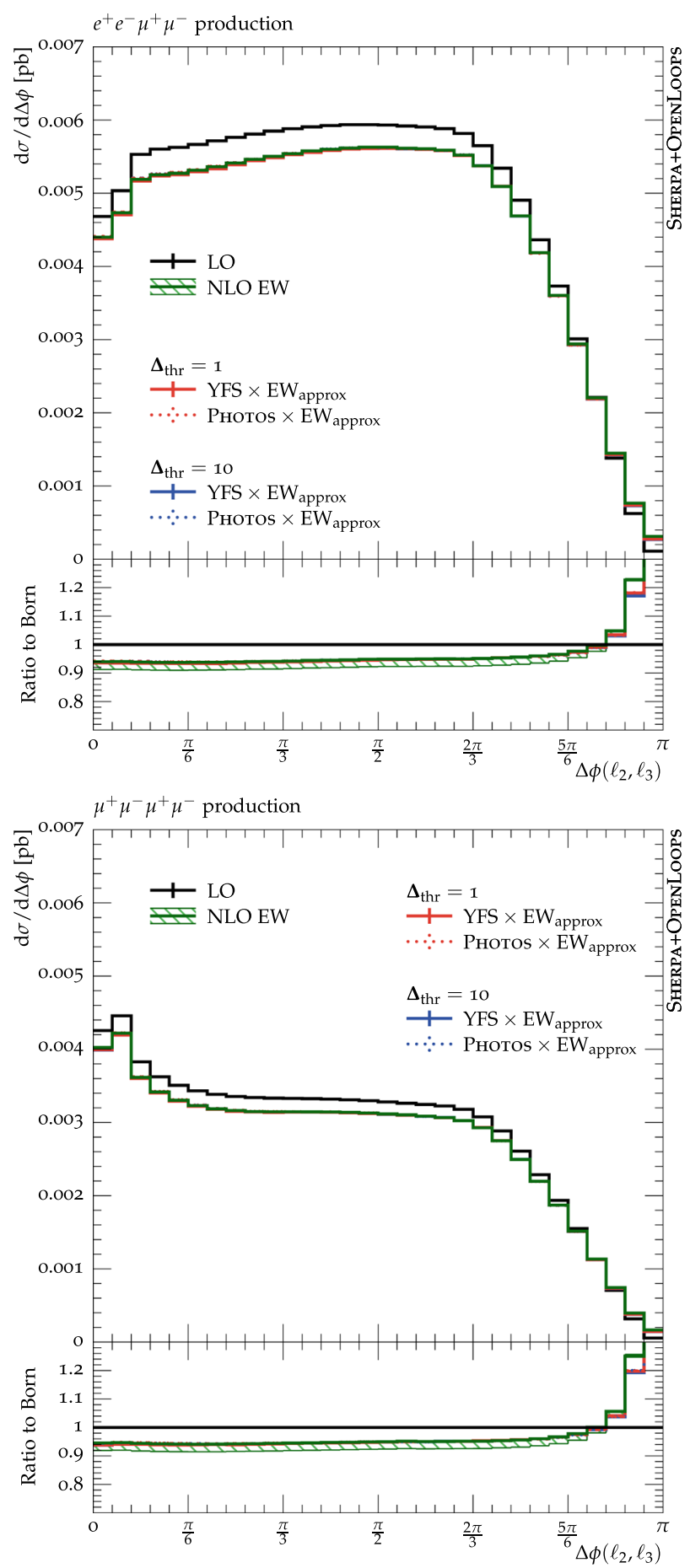

Fig. 12 Differential cross-sections as a function of the azimuthal separation between the $2^{\text {nd }}$ and $3^{\text {rd }}$ leading lepton, $\Delta \phi\left(\ell_{2}, \ell_{3}\right)$, in $e^{+} e^{-} \mu^{+} \mu^{-}$production (top) as well as $\mu^{+} \mu^{-} \mu^{+} \mu^{-}$production (bottom). The NLO EW prediction (green), including its renormalisation scheme uncertainty, is compared to predictions in the $\mathrm{EW}_{\text {approx }}$ approximation, augmented with PHOTOS (dotted) or YFS (solid) using either a

nature of the large corrections indicates, $\mathcal{O}\left(\alpha^{2}\right)$ corrections are expected to be large. This is confirmed by the large deviation the resummations exhibit throughout all dressing-cone
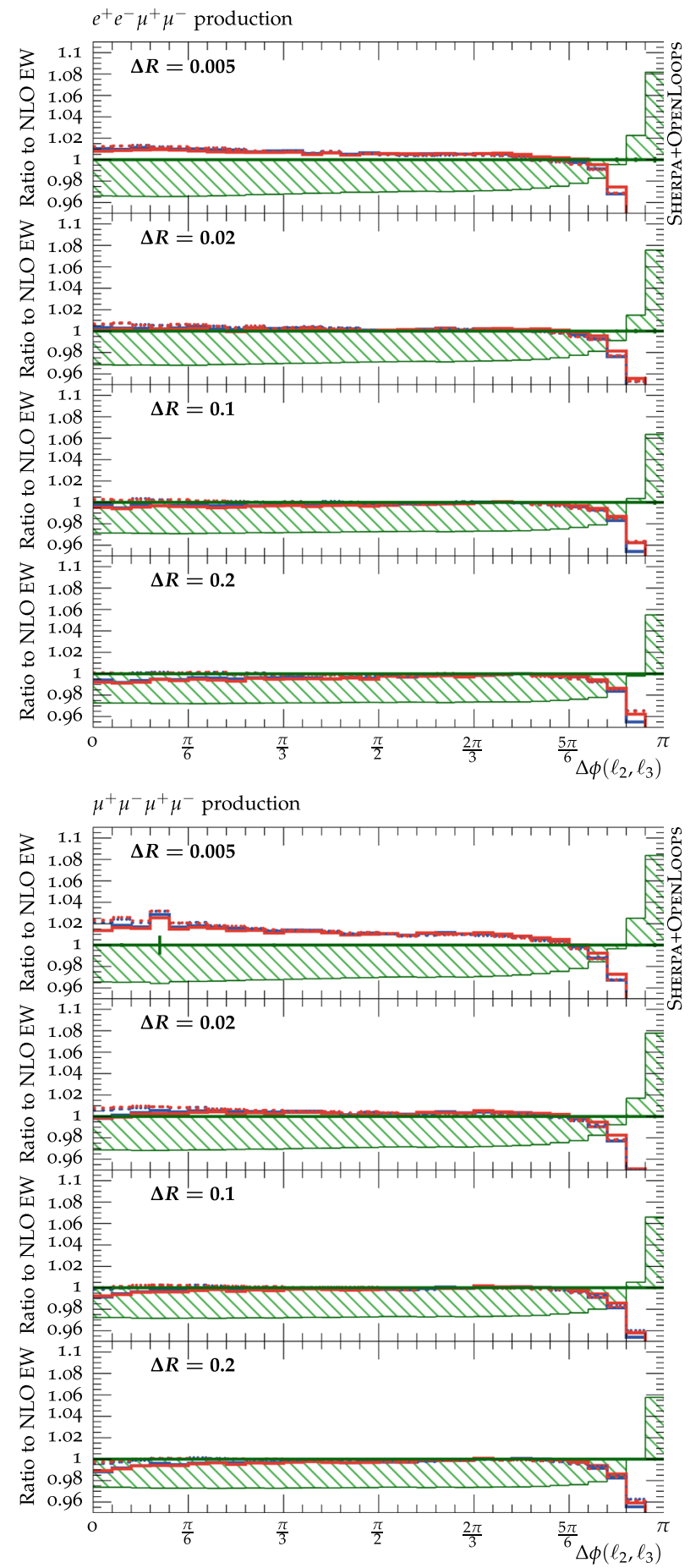

conservative (red) or relaxed (blue) clustering threshold. The Born-level prediction is illustrated by the black curve. The absolute cross-sections are shown on the left for a dressing-cone size of 0.1 , while ratios of the PHOTOS and YFs curves are shown with respect to the NLO EW prediction on the right for different dressing-cone sizes

sizes, being in rather good agreement between themselves. Also in this region, $\Delta_{\mathrm{thr}}$-dependences are small. 

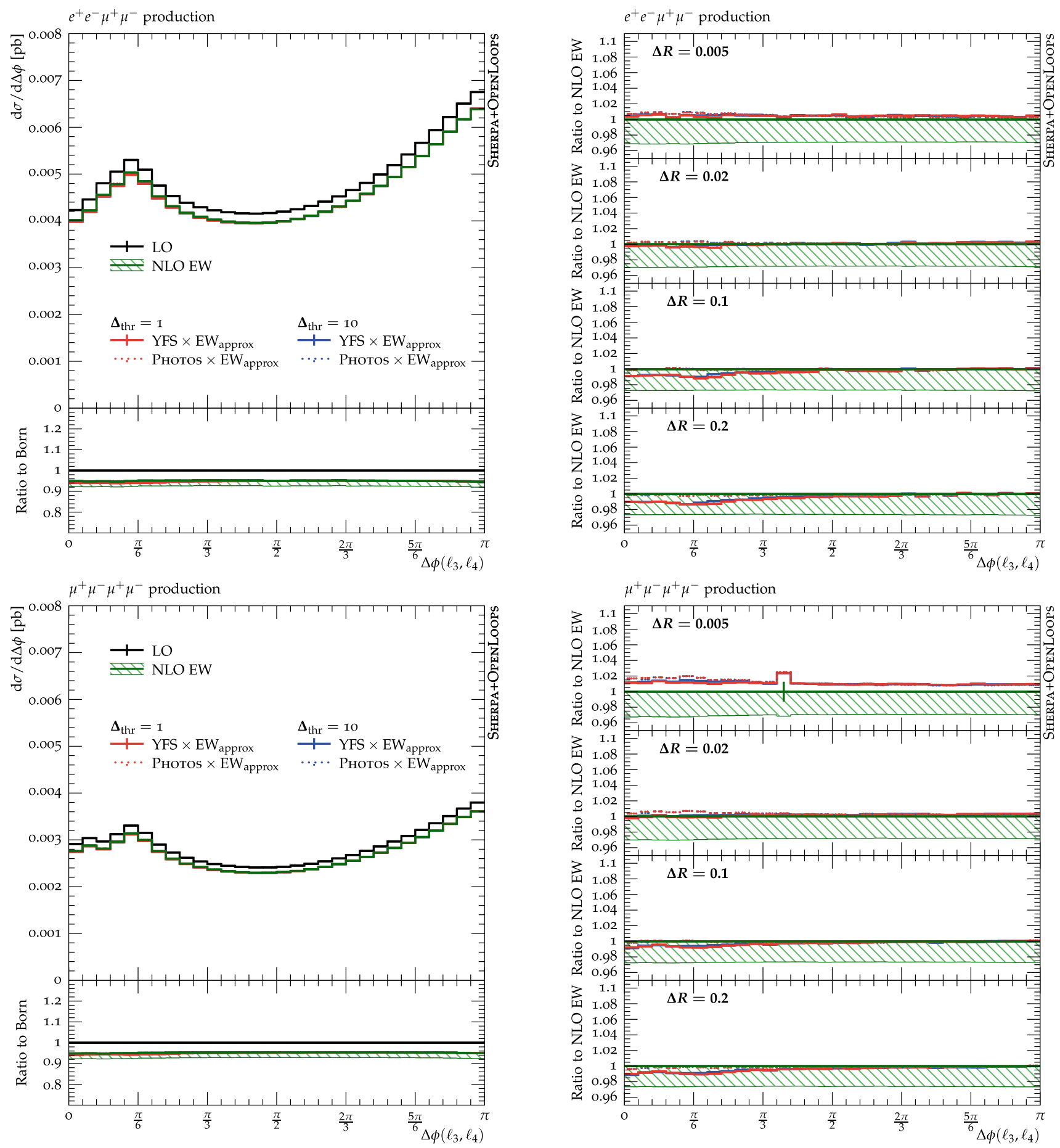

Fig. 13 Differential cross-sections as a function of the azimuthal separation between the $3^{\text {rd }}$ and $4^{\text {th }}$ leading lepton, $\Delta \phi\left(\ell_{3}, \ell_{4}\right)$, in $e^{+} e^{-} \mu^{+} \mu^{-}$production (top) as well as $\mu^{+} \mu^{-} \mu^{+} \mu^{-}$production (bottom). The NLO EW prediction (green), including its renormalisation scheme uncertainty, is compared to predictions in the $\mathrm{EW}_{\text {approx }}$ approximation, augmented with PHOTOS (dotted) or YFS (solid) using either a

Since the first and second lepton are typically in opposite hemispheres, there is a lot of freedom for the orientation of the third lepton. In fact, all $\Delta \phi$ between 0 and $\frac{2 \pi}{3}$ are well

conservative (red) or relaxed (blue) clustering threshold. The Born-level prediction is illustrated by the black curve. The absolute cross-sections are shown on the left for a dressing-cone size of 0.1 , while ratios of the PHOTOS and YFs curves are shown with respect to the NLO EW prediction on the right for different dressing-cone sizes

populated, with exception of the dilepton $\Delta R$ imposed by the selection cut, cf. Fig. 12. The fact that this drop happens at $\Delta R\left(\ell_{2}, \ell_{3}\right)<\frac{\pi}{15} \approx 0.2$ suggests that both leptons are 
not coming from the same $Z$ boson in the different-flavour channel in this region at Born level. In the same-flavour channel, likely due to the presence of a photon-pole between four out of the six lepton-pair combinations, the cross section slightly rises as $\Delta \phi$ tends to zero, until the selection criteria regulate the pole. In turn, the NLO EW corrections and their uncertainties show no shape in this region and reproduce the inclusive corrections. They are, independent of the clustering threshold, also well reproduced by both the NLO $\mathrm{EW}_{\text {approx }} \times Y$ FS and NLO $\mathrm{EW}_{\text {approx }} \times P$ HOTOS approximations, notwithstanding small differences at the level of $1 \%$ in both the same- and different-flavour channel as $\Delta \phi \rightarrow 0$. As before, the agreement with the fixed-order result for large $\Delta R_{\text {dress }}$ is much better than the renormalisation scheme uncertainty, but is worsened for smaller dressing-cone sizes, in line with observations made for earlier observables.

Conversely, the azimuthal difference between the second and the third lepton is suppressed in the back-to-back configuration at $\Delta \phi \approx \pi$. This is again a result of the kinematic suppression of the configurations depicted in Fig. 10d. Photon emissions lift the kinematic restrictions also in this case and allow the second and third lepton closer together, thereby opening up phase space for the back-to-back topology. This is once more manifested as an electroweak enhancement, this time in the region around $\pi$. Both PHOTOS and YFS agree well with one-another, and their difference with fixed-order calculation indicates large $\mathcal{O}\left(\alpha^{2}\right)$ corrections.

For the third and the fourth lepton, the azimuthal difference would be enhanced towards back-to-back or closeby values of $\Delta \phi$. However, the isolation requirements on the leptons suppress the configurations where two of the leptons are very close to each other, giving rise to a kink towards very low values of the azimuthal difference, as can be seen in Fig. 13. No part of the distribution is kinematically suppressed at leading order, hence no region receives large positive radiative corrections. On the contrary, the NLOEW corrections are flat and featureless throughout, and, apart from a $1 \%$ difference between YFS and РнотоS in both the same-flavour and the different-flavour channel for small $\Delta \phi$, are well reproduced by both approximations for inclusive dressing-cone sizes. Virtually no $\Delta_{\text {thr }}$ dependence is observed.

\section{Conclusions}

In this paper we presented a study of kinematic distributions in the four-charged-leptons final state including Born and one-loop EW corrections using the SHERPA and OPENLOOPS frameworks. In addition to the exact NLO EW calculation, we incorparated EW corrections in an approximation, based on exact virtual NLO contributions supplemented with a soft-photon resummation using both РНОTOS as well as SHERPA's soft-photon resummation in the Yennie-Frautschi-
Suura scheme. We showed that this approximation is able to reproduce the full NLO EW result for $p p \rightarrow \ell \ell \ell^{\prime} \ell^{\prime}$ production to within a few percent, which we studied separately for the same-flavour and the different-flavour configuration. We observed that the setup which uses PHOTOS to model the softphoton emissions consistently predicts a larger cross-section than the setup using the YFS scheme, with the largest differences seen in the different-flavour case, while the YFS scheme is generally closer to the fixed-order NLO EW calculation.

We also studied the dependence on the dressing-cone size and find that a cone size of $\Delta R_{\text {dress }}=0.1$ gives the best overall agreement between the two approximations and the fixed-order calculation. Further, while both resummation calculations are expected to give a more reliable dependence on the dressing-cone size $\Delta R_{\text {dress }}$, an adoption of the smallest dressing-cone radius of 0.005 induces both shape- and ratechanges in most distributions. This emphasises the need for a properly matched calculation to combine the resummed description with the formal accuracy of the exact NLO EW calculation. ${ }^{6}$

Finally, we also investigated the effect of the clustering threshold used by SHERPA to preserve resonance structures and observed that, compared to the default value $\Delta_{\mathrm{thr}}=1$, a more relaxed threshold tends to improve the agreement with the fixed-order result in most regions of phase space. This indicates that the QED corrections to the four-lepton final state behave as if the leptons were produced resonantly in a larger region of phase space than a naïve interpretation of the Breit-Wigner width suggests.

Acknowledgements We thank Max Goblirsch-Kolb for many fruitful discussions during early stages of the project. This work has received funding from the European Union's Horizon 2020 research and innovation programme as part of the Marie Skłodowska-Curie Innovative Training Network MCnetITN3 (Grant agreement no. 722104). MS acknowledges the support of the Royal Society (URFR1180549) through the award of a University Research Fellowship.

Data Availability Statement This manuscript has no associated data or the data will not be deposited. [Authors' comment: This a theory calculation. All numbers are contained in the manuscript.]

Open Access This article is licensed under a Creative Commons Attribution 4.0 International License, which permits use, sharing, adaptation, distribution and reproduction in any medium or format, as long as you give appropriate credit to the original author(s) and the source, provide a link to the Creative Commons licence, and indicate if changes were made. The images or other third party material in this article are included in the article's Creative Commons licence, unless indicated otherwise in a credit line to the material. If material is not included in the article's Creative Commons licence and your intended use is not permitted by statutory regulation or exceeds the permitted use, you will need to obtain permission directly from the copyright holder. To view a copy of this licence, visit http://creativecomm

\footnotetext{
6 One such matched calculation has appeared recently in [41] using the PYTHIA8 QED shower.
} 
ons.org/licenses/by/4.0/.

Funded by $\mathrm{SCOAP}^{3}$.

\section{References}

1. J. Brehmer, F. Kling, T. Plehn, T.M.P. Tait, Better Higgs-CP tests through information geometry. Phys. Rev. D 97(9), 095017 (2018). arXiv: 1712.02350 [hep-ph]

2. M. Aaboud et al., ATLAS, measurement of the four-lepton invariant mass spectrum in $13 \mathrm{TeV}$ proton-proton collisions with the ATLAS detector. JHEP 04, 048 (2019). arXiv:1902.05892 [hep-ex]

3. M. Aaboud et al., ATLAS, $Z Z \rightarrow \ell^{+} \ell^{-} \ell^{\prime+} \ell^{\prime-}$ cross-section measurements and search for anomalous triple gauge couplings in 13 $\mathrm{TeV} p p$ collisions with the ATLAS detector. Phys. Rev. D 97(3), 032005 (2018). arXiv:1709.07703 [hep-ex]

4. A.M. Sirunyan et al., CMS, measurements of the $\mathrm{pp} \rightarrow \mathrm{ZZ}$ production cross section and the $\mathrm{Z} \rightarrow 4 \ell$ branching fraction, and constraints on anomalous triple gauge couplings at $\sqrt{s}=13 \mathrm{TeV}$. Eur. Phys. J. C 78, 165 (2018). arXiv:1709.08601 [hep-ex]. (Erratum: Eur. Phys. J. C 78, 515 (2018))

5. G. Aad et al., ATLAS, Measurements of the Higgs boson inclusive and differential fiducial cross sections in the $4 \ell$ decay channel at $\sqrt{s}=13$ TeV. arXiv:2004.03969 [hep-ex]

6. A.M. Sirunyan et al., CMS, measurements of properties of the Higgs boson decaying into the four-lepton final state in pp collisions at $\sqrt{s}=13$ TeV. JHEP 11, 047 (2017). arXiv:1706.09936 [hep-ex]

7. F.U. Bernlochner, C. Englert, C. Hays, K. Lohwasser, H. Mildner, A. Pilkington, D.D. Price, M. Spannowsky, Angles on CP-violation in Higgs boson interactions. Phys. Lett. B 790, 372-379 (2019). arXiv:1808.06577 [hep-ph]

8. ATLAS, Constraints on the Higgs boson self-coupling from the combination of single-Higgs and double-Higgs production analyses performed with the ATLAS experiment, ATLAS-CONF-2019049

9. J. Ohnemus, J. Owens, An Order $\alpha_{s}$ calculation of hadronic $Z Z$ production. Phys. Rev. D 43, 3626-3639 (1991). (FSU-HEP901212)

10. B. Mele, P. Nason, G. Ridolfi, QCD radiative corrections to Z boson pair production in hadronic collisions. Nucl. Phys. B 357, 409-438 (1991). (CERN-TH-5890-90, GEF-TH-17-1990, UPRF-90-20)

11. J.M. Campbell, R. Ellis, An update on vector boson pair production at hadron colliders. Phys. Rev. D 60, 113006 (1999). arXiv:hep-ph/9905386

12. L.J. Dixon, Z. Kunszt, A. Signer, Vector boson pair production in hadronic collisions at order $\alpha_{s}$ : lepton correlations and anomalous couplings. Phys. Rev. D 60, 114037 (1999). arXiv:hep-ph/9907305

13. F. Cascioli, T. Gehrmann, M. Grazzini, S. Kallweit, P. Maierhöfer, A. von Manteuffel, S. Pozzorini, D. Rathlev, L. Tancredi, E. Weihs, ZZ production at hadron colliders in NNLO QCD. Phys. Lett. B 735, 311-313 (2014). arXiv:1405.2219 [hep-ph]

14. M. Grazzini, S. Kallweit, D. Rathlev, ZZ production at the LHC: fiducial cross sections and distributions in NNLO QCD. Phys. Lett. B 750, 407-410 (2015). arXiv:1507.06257 [hep-ph]

15. S. Kallweit, M. Wiesemann, $Z Z$ production at the LHC: NNLO predictions for $2 \ell 2 v$ and $4 \ell$ signatures. Phys. Lett. B 786, 382-389 (2018). arXiv:1806.05941 [hep-ph]

16. D.A. Dicus, C. Kao, W. Repko, Gluon production of gauge bosons. Phys. Rev. D 36, 1570 (1987). (DOE-ER-40200-100)

17. E. Glover, J. van der Bij, $Z$ boson pair production via gluon fusion. Nucl. Phys. B 321, 561-590 (1989). (CERN-TH-5248/88)

18. T. Matsuura, J. van der Bij, Characteristics of leptonic signals for Z boson pairs at hadron colliders. Z. Phys. C 51, 259-266 (1991). (DESY-91-004)
19. C. Zecher, T. Matsuura, J. van der Bij, Leptonic signals from offshell Z boson pairs at hadron colliders. Z. Phys. C 64, 219-226 (1994). arXiv:hep-ph/9404295

20. F. Caola, K. Melnikov, R. Röntsch, L. Tancredi, QCD corrections to ZZ production in gluon fusion at the LHC. Phys. Rev. D 92(9), 094028 (2015). arXiv:1509.06734 [hep-ph]

21. F. Caola, M. Dowling, K. Melnikov, R. Röntsch, L. Tancredi, QCD corrections to vector boson pair production in gluon fusion including interference effects with off-shell Higgs at the LHC. JHEP 07, 087 (2016). arXiv: 1605.04610 [hep-ph]

22. M. Grazzini, S. Kallweit, M. Wiesemann, J.Y. Yook, $Z Z$ production at the LHC: NLO QCD corrections to the loop-induced gluon fusion channel. JHEP 03, 070 (2019). arXiv:1811.09593 [hep-ph]

23. P. Nason, G. Ridolfi, A positive-weight next-to-leading-order Monte Carlo for Z pair hadroproduction. JHEP 08, 077 (2006). arXiv:hep-ph/0606275

24. K. Hamilton, A positive-weight next-to-leading order simulation of weak boson pair production. JHEP 01, 009 (2011). arXiv:1009.5391 [hep-ph]

25. S. Höche, F. Krauss, M. Schönherr, F. Siegert, Automating the POWHEG method in Sherpa. JHEP 04, 024 (2011). arXiv:1008.5399 [hep-ph]

26. T. Melia, P. Nason, R. Röntsch, G. Zanderighi, W+W-, WZ and ZZ production in the POWHEG BOX. JHEP 11, 078 (2011). arXiv:1107.5051 [hep-ph]

27. R. Frederix, S. Frixione, V. Hirschi, F. Maltoni, R. Pittau, P. Torrielli, Four-lepton production at hadron colliders: aMC@NLO predictions with theoretical uncertainties. JHEP 02, 099 (2012). arXiv: 1110.4738 [hep-ph]

28. S. Alioli, F. Caola, G. Luisoni, R. Röntsch, ZZ production in gluon fusion at NLO matched to parton-shower. Phys. Rev. D 95(3), 034042 (2017). arXiv:1609.09719 [hep-ph]

29. W. Beenakker, A. Denner, S. Dittmaier, R. Mertig, T. Sack, Highenergy approximation for on-shell $\mathrm{W}$ pair production. Nucl. Phys. B 410, 245-279 (1993). (CERN-TH-6832-93)

30. M. Beccaria, G. Montagna, F. Piccinini, F. Renard, C. Verzegnassi, Rising bosonic electroweak virtual effects at high-energy $e^{+} e^{-}$ colliders. Phys. Rev. D 58, 093014 (1998). arXiv:hep-ph/9805250

31. P. Ciafaloni, D. Comelli, Sudakov enhancement of electroweak corrections. Phys. Lett. B 446, 278-284 (1999). arXiv:hep-ph/9809321

32. J.H. Kühn, A. Penin, Sudakov logarithms in electroweak processes. arXiv:hep-ph/9906545

33. V.S. Fadin, L.N. Lipatov, A.D. Martin, M. Melles, Resummation of double logarithms in electroweak high-energy processes. Phys. Rev. D 61, 094002 (2000). arXiv:hep-ph/9910338

34. A. Denner, S. Pozzorini, One loop leading logarithms in electroweak radiative corrections. 1. Results. Eur. Phys. J. C 18, 461480 (2001). arXiv:hep-ph/0010201

35. E. Accomando, A. Denner, A. Kaiser, Logarithmic electroweak corrections to gauge-boson pair production at the LHC. Nucl. Phys. B 706, 325-371 (2005). arXiv:hep-ph/0409247

36. A. Bierweiler, T. Kasprzik, J.H. Kühn, Vector-boson pair production at the LHC to $\mathcal{O}\left(\alpha^{3}\right)$ accuracy. JHEP 12, 071 (2013). arXiv:1305.5402 [hep-ph]

37. J. Baglio, L.D. Ninh, M.M. Weber, Massive gauge boson pair production at the LHC: a next-to-leading order story. Phys. Rev. D 88, 113005 (2013). arXiv:1307.4331 [hep-ph]. (Erratum: Phys. Rev. D 94, 099902 (2016))

38. B. Biedermann, A. Denner, S. Dittmaier, L. Hofer, B. Jäger, Electroweak corrections to $p p \rightarrow \mu^{+} \mu^{-} e^{+} e^{-}+X$ at the LHC: a Higgs background study. Phys. Rev. Lett. 116(16), 161803 (2016). arXiv:1601.07787 [hep-ph]

39. B. Biedermann, A. Denner, S. Dittmaier, L. Hofer, B. Jäger, Next-to-leading-order electroweak corrections to the production 
of four charged leptons at the LHC. JHEP 01, 033 (2017). arXiv:1611.05338 [hep-ph]

40. M. Grazzini, S. Kallweit, J.M. Lindert, S. Pozzorini, M. Wiesemann, NNLO QCD + NLO EW with Matrix + OpenLoops: precise predictions for vector-boson pair production. JHEP 02, 087 (2020). arXiv:1912.00068 [hep-ph]

41. M. Chiesa, C. Oleari, E. Re, NLO QCD+NLO EW corrections to diboson production matched to parton shower. arXiv:2005.12146 [hep-ph]

42. F. Cascioli, S. Höche, F. Krauss, P. Maierhöfer, S. Pozzorini, F. Siegert, Precise Higgs-background predictions: merging NLO QCD and squared quark-loop corrections to four-lepton $+0,1$ jet production. JHEP 01, 046 (2014). arXiv: 1309.0500 [hep-ph]

43. M.H. Seymour, Photon radiation in final state parton showering. Z. Phys. C 56, 161-170 (1992). (CAVENDISH-HEP-91-16)

44. S. Höche, S. Schumann, F. Siegert, Hard photon production and matrix-element parton-shower merging. Phys. Rev. D 81, 034026 (2010). arXiv:0912.3501 [hep-ph]

45. J. Bellm et al., Herwig 7.2 release note. Eur. Phys. J. C 80(5), 452 (2020). arXiv:1912.06509 [hep-ph]

46. T. Sjöstrand, S. Ask, J.R. Christiansen, R. Corke, N. Desai, P. Ilten, S. Mrenna, S. Prestel, C.O. Rasmussen, P.Z. Skands, An introduction to PYTHIA 8.2. Comput. Phys. Commun. 191, 159-177 (2015). arXiv:1410.3012 [hep-ph]

47. E. Bothmann et al., Event generation with SHERPA 2, 2 (2019)

48. E. Barberio, B. van Eijk, Z. Was, PHOTOS: a universal Monte Carlo for QED radiative corrections in decays. Comput. Phys. Commun. 66, 115-128 (1991). (CERN-TH-5857-90)

49. C.M. Carloni Calame, An improved parton shower algorithm in QED. Phys. Lett. B 520, 16-24 (2001). arXiv:hep-ph/0103117

50. K. Hamilton, P. Richardson, Simulation of QED radiation in particle decays using the YFS formalism. JHEP 07, 010 (2006). arXiv:hep-ph/0603034

51. M. Schönherr, F. Krauss, Soft photon radiation in particle decays in SHERPA. JHEP 12, 018 (2008). arXiv:0810.5071 [hep-ph]

52. S. Gieseke, T. Kasprzik, J.H. Kühn, Vector-boson pair production and electroweak corrections in HERWIG++. Eur. Phys. J. C 74(8), 2988 (2014). arXiv:1401.3964 [hep-ph]

53. E. Bothmann, D. Napoletano, Automated evaluation of electroweak Sudakov logarithms in Sherpa. arXiv:2006.14635 [hep-ph]

54. S. Kallweit, J.M. Lindert, P. Maierhöfer, S. Pozzorini, M. Schönherr, NLO QCD + EW predictions for V + jets including off-shell vector-boson decays and multijet merging. JHEP 04, 021 (2016). arXiv:1511.08692 [hep-ph]

55. D.R. Yennie, S.C. Frautschi, H. Suura, The infrared divergence phenomena and high-energy processes. Ann. Phys. 13, 379-452 (1961)

56. T. Gleisberg, S. Höche, F. Krauss, M. Schönherr, S. Schumann, F. Siegert, J. Winter, Event generation with SHERPA 1.1. JHEP 02, 007 (2009). arXiv:0811.4622 [hep-ph]

57. F. Buccioni, J.-N. Lang, J.M. Lindert, P. Maierhöfer, S. Pozzorini, H. Zhang, M.F. Zoller, OpenLoops 2. Eur. Phys. J. C 79(10), 866 (2019). arXiv:1907.13071 [hep-ph]

58. F. Cascioli, P. Maierhöfer, S. Pozzorini, Scattering amplitudes with open loops. Phys. Rev. Lett. 108, 111601 (2012). arXiv:1111.5206 [hep-ph]

59. A. Denner, S. Dittmaier, L. Hofer, Collier: a fortran-based complex one-loop LIbrary in extended regularizations. Comput. Phys. Commun. 212, 220-238 (2017). arXiv:1604.06792 [hep-ph]

60. G. Ossola, C.G. Papadopoulos, R. Pittau, CutTools: a program implementing the OPP reduction method to compute one-loop amplitudes. JHEP 03, 042 (2008). arXiv:0711.3596 [hep-ph]

61. A. van Hameren, OneLOop: for the evaluation of one-loop scalar functions. Comput. Phys. Commun. 182, 2427-2438 (2011). arXiv:1007.4716 [hep-ph]
62. F. Krauss, R. Kuhn, G. Soff, AMEGIC++ 1.0: a matrix element generator in C++. JHEP 02, 044 (2002). arXiv:hep-ph/0109036 [hep-ph]

63. M. Schönherr, An automated subtraction of NLO EW infrared divergences. Eur. Phys. J. C 78(2), 119 (2018). arXiv:1712.07975 [hep-ph]

64. T. Gleisberg, F. Krauss, Automating dipole subtraction for QCD NLO calculations. Eur. Phys. J. C 53, 501-523 (2008). arXiv:0709.2881 [hep-ph]

65. S. Kallweit, J.M. Lindert, P. Maierhöfer, S. Pozzorini, M. Schönherr, NLO electroweak automation and precise predictions for $\mathrm{W}+$ multijet production at the LHC. JHEP 04, 012 (2015). arXiv: 1412.5157 [hep-ph]

66. S. Kallweit, J.M. Lindert, S. Pozzorini, M. Schönherr, NLO QCD+EW predictions for $2 \ell 2 v$ diboson signatures at the LHC. JHEP 11, 120 (2017). arXiv:1705.00598 [hep-ph]

67. B. Biedermann, S. Bräuer, A. Denner, M. Pellen, S. Schumann, J.M. Thompson, Automation of NLO QCD and EW corrections with Sherpa and Recola. Eur. Phys. J. C 77, 492 (2017). arXiv: 1704.05783 [hep-ph]

68. J.M. Lindert et al., Precise predictions for $V+$ jets dark matter backgrounds. Eur. Phys. J. C 77(12), 829 (2017). arXiv:1705.04664 [hep-ph]

69. M. Chiesa, N. Greiner, M. Schönherr, F. Tramontano, Electroweak corrections to diphoton plus jets. JHEP 10, 181 (2017). arXiv:1706.09022 [hep-ph]

70. N. Greiner, M. Schönherr, NLO QCD + EW corrections to diphoton production in association with a vector boson. JHEP 01, 079 (2018). arXiv:1710.11514 [hep-ph]

71. C. Gütschow, J.M. Lindert, M. Schönherr, Multi-jet merged toppair production including electroweak corrections. Eur. Phys. J. C 78(4), 317 (2018). arXiv:1803.00950 [hep-ph]

72. M. Schönherr, Next-to-leading order electroweak corrections to off-shell WWW production at the LHC. JHEP 07, 076 (2018). arXiv:1806.00307 [hep-ph]

73. M. Reyer, M. Schönherr, S. Schumann, Full NLO corrections to 3 -jet production and $R_{32}$ at the LHC. Eur. Phys. J. C 79(4), 321 (2019). arXiv:1902.01763 [hep-ph]

74. S. Bräuer, A. Denner, M. Pellen, M. Schönherr, S. Schumann, Fixed-order and merged parton-shower predictions for WW and WWj production at the LHC including NLO QCD and EW corrections. arXiv:2005.12128 [hep-ph]

75. Les Houches, Physics at TeV colliders standard model working group report 3, 2018 (2017)

76. E. Barberio, Z. Was, PHOTOS: a universal Monte Carlo for QED radiative corrections. Version 2.0. Comput. Phys. Commun. 79, 291-308 (1994). (CERN-TH-7033-93)

77. P. Golonka, Z. Was, PHOTOS Monte Carlo: a precision tool for QED corrections in $Z$ and $W$ decays. Eur. Phys. J. C 45, 97-107 (2006). arXiv:hep-ph/0506026

78. N. Davidson, T. Przedzinski, Z. Was, PHOTOS interface in C++: technical and physics documentation. Comput. Phys. Commun. 199, 86-101 (2016). arXiv:1011.0937 [hep-ph]

79. F. Krauss, J.M. Lindert, R. Linten, M. Schönherr, Accurate simulation of W, Z and Higgs boson decays in Sherpa. Eur. Phys. J. C 79(2), 143 (2019). arXiv:1809.10650 [hep-ph]

80. R.D. Ball et al., NNPDF. Parton distributions for the LHC Run II. JHEP 04, 040 (2015). arXiv:1410.8849 [hep-ph]

81. A. Buckley, J. Ferrando, S. Lloyd, K. Nordström, B. Page, M. Rüfenacht, M. Schönherr, G. Watt, LHAPDF6: parton density access in the LHC precision era. Eur. Phys. J. C 75(3), 132 (2015). arXiv: 1412.7420 [hep-ph]

82. C. Bierlich et al., Robust independent validation of experiment and theory: Rivet version 3. Sci. Post Phys. 8, 026 (2020). arXiv:1912.05451 [hep-ph] 